\title{
Standard model EFT effects in vector-boson scattering at the LHC
}

\author{
A. Dedes, ${ }^{1}$ P. Kozów $\odot,{ }^{2}$ and M. Szleper ${ }^{3}$ \\ ${ }^{1}$ Department of Physics, Division of Theoretical Physics, University of Ioannina, GR 45110, Greece \\ ${ }^{2}$ Institute of Theoretical Physics, Faculty of Physics, University of Warsaw, \\ ul. Pasteura 5, 02-093 Warsaw, Poland \\ ${ }^{3}$ National Center for Nuclear Research, High Energy Physics Department, \\ ul. Pasteura 7, 02-093 Warsaw, Poland
}

(Received 23 February 2021; accepted 15 June 2021; published 13 July 2021)

\begin{abstract}
The assumption that the Standard Model is an effective field theory (SM EFT) of a more fundamental theory at a higher (than electroweak) energy scale implies a growth of cross sections for electroweak vector-boson scattering (VBS) processes signaling the appearance of a resonance (or resonances) near that scale. In this article, we investigate in detail SM EFT effects from dimension-six operators in VBS with like-sign- $W$ production in fully leptonic decay modes at the high-luminosity LHC (HL-LHC). We find that these effects are important for a handful of operators, most notably for the operator composed of three $S U$ (2) field-strength tensors responsible for strong transversely polarized vector-boson interactions. Current global fits on Wilson coefficients allow for an observable signal at the HL-LHC, if not accessible with the current LHC data set.
\end{abstract}

DOI: 10.1103/PhysRevD.104.013003

\section{INTRODUCTION}

It is widely believed that the Standard Model (SM) [1-3] is an effective, low-energy field theory (EFT) approximation [4-6] to a more fundamental theory (UV theory) which overtakes the SM at energies (at least several times) higher than the electroweak (EW) scale. One of the most important processes that may shed light on the dynamics of the UV theory is electroweak vector-boson scattering (VBS). ${ }^{1}$ This claim is true for the following reasons:

(i) VBS processes are directly related to the mechanism of electroweak symmetry breaking, and as such are regarded as complementary to Higgs boson measurements at the LHC.

(ii) Although inaccessible directly, heavy particles (constituents of the UV theory) may leave a trace by modifying the strength and dynamics of electroweak interactions, spoiling, for example, the (at most constant) center-of-mass energy $(s)$ behavior of the SM $V V \rightarrow V V\left(V=W^{ \pm}, Z, \gamma\right)$ amplitudes. In the SM EFT, this results in a growth of cross sections at energies immediately after the electroweak scale.

\footnotetext{
${ }^{1}$ For a review, see Ref. [7].
}

Published by the American Physical Society under the terms of the Creative Commons Attribution 4.0 International license. Further distribution of this work must maintain attribution to the author(s) and the published article's title, journal citation, and DOI. Funded by SCOAP. (iii) In the SM, even when next-to-leading-order corrections are included, VBS processes feature a particularly slow slope with energy in comparison to other electroweak processes [8].

Therefore, points (ii) and (iii) imply potential sensitivity to the UV theory particles, especially to those strongly interacting with EW gauge bosons.

Unfortunately, there is no $V V$ collider available ${ }^{2}$; however, the $V V \rightarrow V V$ reactions are indirectly accessible at the LHC, particularly within its high-luminosity phase (HL-LHC), through the process $p p \rightarrow 2$ jets +2 lepton pairs. While all of the elastic processes, e.g., $Z Z, W Z$, $W^{ \pm} W^{ \pm}, W^{ \pm} W^{\mp}$ have been extensively investigated at the LHC, the same-sign $W^{+} W^{+}$process in the fully leptonic decay mode was the first process to be observed ${ }^{3}$ at $5 \sigma$ [12] (based on the $13 \mathrm{TeV}$ data set), and is currently confirmed at a sensitivity far above $5 \sigma$ [11]. For that reason, in this paper we investigate in detail the VBS process through $W^{+} W^{+}$scattering, namely, the reaction

$$
\begin{aligned}
p p & \rightarrow 2 \text { jets }+W^{+*} W^{+*} \\
& \rightarrow 2 \text { jets }+2 \text { charged-leptons }+2 \text { neutrinos. }
\end{aligned}
$$

This is exemplified by Fig. 1 for a particular final state. Throughout this work, we mostly focus on the HL-LHC experimental perspectives.

\footnotetext{
${ }^{2}$ Interestingly, in a certain setup it has been argued that a muon collider could effectively be regarded as such $[9,10]$.

${ }^{3}$ The actual measurement involves a sum of both $W^{+} W^{+}$and $W^{-} W^{-}$processes; $W^{+} W^{+}$makes up about $4 / 5$ of it [11].
} 


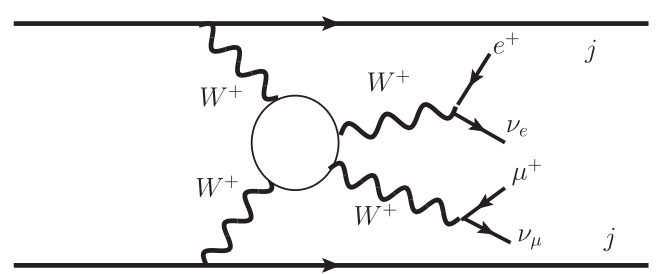

FIG. 1. Feynman diagram depicting a VBS process.

We follow the SM EFT approach ${ }^{4}$ in which the corrections from heavy and decoupled UV states [14] can be parametrized by a number of $i$-indexed dimension-six operators $Q_{i}$ added to the SM Lagrangian, associated with dimensionless (or dimensionful) Wilson coefficients $C^{i}\left(\right.$ or $\left.f^{i}\right)$ as

$$
\mathcal{L}=\mathcal{L}_{\mathrm{SM}}+\sum_{i} \frac{C^{i}}{\Lambda^{2}} Q_{i}+\ldots \equiv \mathcal{L}_{\mathrm{SM}}+\sum_{i} f^{i} Q_{i}+\ldots
$$

The mass scale $\Lambda$ denotes the lightest mass among the heavy particle masses within the UV theory. Although the EFT expansion in Eq. (1.1) is written up to $1 / \Lambda^{2}$, in one occasion below we will fill in the "..." with several dimension-eight operators where the dimensionless Wilson coefficients are suppressed by four powers of $\Lambda$ 's.

The real advantage of the EFT approach follows from the fact that one can study the discovery potential of physics beyond the SM (BSM) without any knowledge of theories that may lie ahead by making use of a limited number of operators arranged order by order in a $1 / \Lambda$ expansion. More precisely, here we consider the SM EFT theory that matches UV models featuring linearly realized electroweak symmetry breaking, where the Higgs field is part of the SM Higgs doublet. The SM EFT basis of nonredundant operators was first constructed at dimension six (commonly referred to as the Warsaw basis [15]) and very recently at dimension eight $[16,17]$.

It is a common approach in both experimental and theoretical literature to use EFT to study VBS channels as an indirect search for BSM physics [18-22]; for a recent review, see Ref. [23]. The point of focus of such studies is usually the $V V V V$ quartic couplings, i.e., the operators that modify quartic gauge couplings (QGCs) and simultaneously leave intact trilinear gauge couplings (TGCs) and Higgs-gauge boson interactions. In SM EFT such kind of physics arise from dimension-eight operators [24,25] and, consequently, the searches are typically conducted as if there was no effect arising from dimension-six operators. Here we investigate the validity of this assumption, given experimental constraints on dimension-six interactions (which necessarily modify TGCs) emerging from independent channels, by studying in detail the like-sign $W$-boson

\footnotetext{
${ }^{4}$ For a review see, Ref. [13].
}

production through VBS at the (HL-)LHC. We would like to emphasize that the aim of this work is not to accurately model possible BSM signals, but to check which dimension-six operators can produce non-negligible effect as allowed by the most up to date experimental constraints from non-VBS processes on the corresponding Wilson coefficients (including also the important issue of bounding background operators), which include new LHC Run 2 results. The numerical significance of dimension-six operators in VBS has been pointed out in Refs. [26,27].

Concerning the bounds on dimension-six operators, we use the ones reported in Refs. [28,29] (non-four-fermion operators) and [30,31] (four-fermion operators). ${ }^{5}$ In Refs. [28,29], the truncation of EFT cross sections was performed consistently at dimension six, namely, no $(\text { dimension-six })^{2}$ terms were considered that would arise from squaring the $(\mathrm{SM}+$ dimension-six $)$ amplitude. It is known that the inclusion of the latter significantly improves the constraints from diboson production channels. However, dimension-eight effects in the EFT expansion are then generally expected to be significant as well, if the underlying UV interactions are not particularly strong. Therefore, the inclusion of (dimension-six) ${ }^{2}$ terms in principle implies losing model independence within EFT [33]. On the other hand, there is a certain sensitivity to the dimension-six Wilson coefficients in the cross sections and the reported limits are, therefore, to be understood as conservative constraints.

There are many LHC analyses, a partial list being Refs. [11,12,34,35]. Thus far, many $V V \rightarrow V V$ processes have been discovered, but within errors they agree with the SM. Interestingly, current experimental precision does not constrain the "weak coupling" regime, leaving plenty of space for new physics effects. It is particularly attractive since the uncertainties after LHC Run 2 are (by far) "statistics dominated" [11].

The paper is organized as follows. In Sec. II we present the relevant EFT operators and, using analytical formulas from the Appendix, theoretically motivate the more technical subsequent analysis. The core numerical analysis for VBS at the (HL-)LHC is presented in Sec. III. We conclude with Sec. IV.

\section{WARMING UP: $W^{+} W^{+} \rightarrow W^{+} W^{+}$SCATTERING}

As we mentioned in the Introduction, although LHC experimental analyses of VBS are optimized for QGCs (dimension-eight operators), it is important, if not necessary, to examine the impact of TGCs (dimension-six operators) on "golden" process $W^{+} W^{+} \rightarrow W^{+} W^{+}$. After all, dimension-six operators arise at leading order in the EFT expansion. At tree level in SM EFT, as we prove

\footnotetext{
${ }^{5}$ Notice, however, the discussion in Ref. [32].

${ }^{6}$ In Sec. III we also examine the effect of "background" dimension-six operators, which affect other subprocesses.
} 

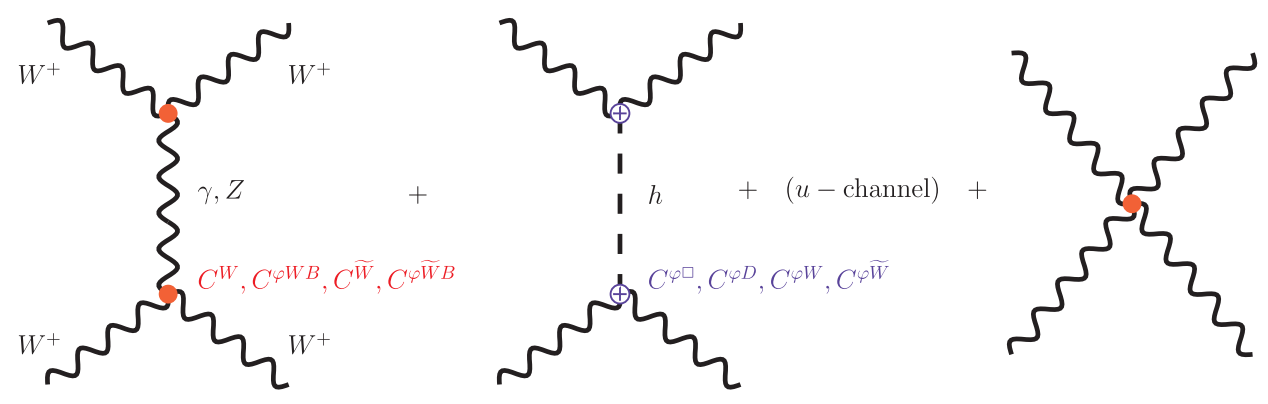

FIG. 2. Tree-level Feynman diagrams filling in the white blob of Fig. 1. The Wilson coefficients associated with the operators affecting each vertex are also shown.

explicitly, the process $W^{+} W^{+} \rightarrow W^{+} W^{+}$is gauge invariant, that is, independent of the gauge-fixing parameter, and hence its self-study is well defined. In both the SM and SM EFT at leading order there are seven Feynman diagrams, shown in Fig. 2, mediated by a photon, a $Z$ boson, and a Higgs boson in both the $t$ and $u$ channels, plus a contact diagram. Following closely the Warsaw basis notation [15], the Lagrangian (1.1) is affected at tree level by five $C P$-conserving $(\mathrm{CPC})$ and three $C P$-violating (CPV) dimension-six operators, shown in Table I.

The leading high-energy helicity amplitudes are given in the Appendix. We work in the Warsaw basis and use the Feynman rules in $R_{\xi}$ gauges from Ref. [36]. The operators $Q_{\varphi W B}$ and $Q_{\varphi \tilde{W} B}$ disappear from the leading-s helicity amplitudes in $W W \rightarrow W W$ processes. This is easily seen by looking at the SM EFT Feynman rules and using the Goldstone boson equivalence theorem [37-43], where for these operators (and specifically the $W W \rightarrow W W$ channel) there are no associated contact four-point interactions involving Goldstone bosons or contact interactions with four W's proportional to $C^{\varphi W B}$ or $C^{\varphi \tilde{W} B}$. One may think that there may be contributions from gluing the three-point vector-boson vertices shown in Fig. 2 where these insertions exist, but it can be proved explicitly that any $s$-enhanced amplitude cancels out when consistently expanding the $Z$ mass, the latter being also affected by $C_{\varphi W B}$. Another important feature is the appearance of the $t$-channel enhancement $\left(1-\cos ^{2} \theta\right)$, where $\theta$ is the scattering angle, in the denominator of leading SM amplitudes; see, for example, $\mathcal{M}_{++++}$in Eq. (A11). This is in contrast to the fact that none of the SM EFT amplitudes have such a $t$-channel enhanced factor that is accompanied by a growth of energy; see Eqs. (A17)-(A25).

Furthermore, in Appendix we also arrange analytical expressions for the helicity cross sections, following the notation where $T$ stands for transverse helicities \pm 1 and $L$ stands for longitudinal gauge bosons with helicity 0 . In the $\mathrm{SM}$, the dominant polarized cross sections come with the following ratios:

$$
\begin{aligned}
& \sigma_{T T T T}: \sigma_{L L L L}: \sigma_{L T L T}: \sigma_{T L T L}: \sigma_{T L L T}: \sigma_{L T T L} \\
& \quad \approx 1: \frac{1}{8.5}: \frac{1}{8.0}: \frac{1}{8.0}: \frac{1}{8.0}: \frac{1}{8.0} .
\end{aligned}
$$

As we already mentioned, in the SM all polarized cross sections are enhanced by the $t$-channel factor, but for the TTTT mode there is an accidentally enhanced factor of 8 with respect to the other modes, as can easily be seen from Eqs. (A26)-(A28).

In SM EFT there are interference effects between the SM and dimension-six operators only in the $L L L L$ mode (or " $0000 "$ mode). $C P$-violating contributions enter in the mixed and pure transverse channels, similar to their $C P$-conserving counterparts. The cross sections have the symbolic form $\sigma \sim \mathrm{SM}^{2}+\mathrm{SM} \times \operatorname{dim} 6+\operatorname{dim} 6^{2}$. Following this pattern, we obtain $\left[\bar{g}^{2}\right.$ is the $S U(2)_{L}$ gauge coupling]

$$
\begin{aligned}
\sigma_{T T T T}(s) \approx & \frac{\bar{g}^{4}}{s}\left[\frac{A_{T}}{1-c^{2}}+B_{T} \cdot 0\right. \\
& \left.+\Gamma_{T} \bar{g}^{2}\left(\frac{\left|C^{W}\right|}{\bar{g}^{2}}\right)^{2}\left(\frac{s}{\Lambda^{2}}\right)^{2}+\cdots\right],
\end{aligned}
$$

TABLE I. Dimension-six operators, in the Warsaw basis, modifying the process $W^{+} W^{+} \rightarrow W^{+} W^{+}$.

\begin{tabular}{llll}
\hline \hline & $X^{3}$ & \multicolumn{1}{c}{$\varphi^{4} D^{2}$} & \multicolumn{1}{c}{$X^{2} \varphi^{2}$} \\
\hline $\mathrm{CPC}$ & $Q_{W}=\epsilon^{I J K} W_{\mu}^{\nu I} W_{\nu}^{\rho J} W_{\rho}^{\mu K}$ & $Q_{\varphi \square}=\left(\varphi^{\dagger} \varphi\right) \square\left(\varphi^{\dagger} \varphi\right)$ & $Q_{\varphi W}=\varphi^{\dagger} \varphi W_{\mu \nu}^{I} W^{\mu \nu I}$ \\
& & $Q_{\varphi D}=\left(\varphi^{\dagger} D^{\mu} \varphi\right)^{*}\left(\varphi^{\dagger} D_{\mu} \varphi\right)$ & $Q_{\varphi W B}=\varphi^{\dagger} \tau^{I} \varphi W_{\mu \nu}^{I} B^{\mu \nu}$ \\
$\mathrm{CPV}$ & $Q_{\tilde{W}}=\epsilon^{I J K} \tilde{W}_{\mu}^{\nu I} W_{\nu}^{\rho J} W_{\rho}^{\mu K}$ & & $Q_{\varphi \tilde{W}}=\varphi^{\dagger} \varphi \tilde{W}_{\mu \nu}^{I} W^{\mu \nu I}$ \\
& & $Q_{\varphi \tilde{W} B}=\varphi^{\dagger} \tau^{I} \varphi \tilde{W}_{\mu \nu}^{I} W^{\mu \nu I}$ \\
\hline \hline
\end{tabular}



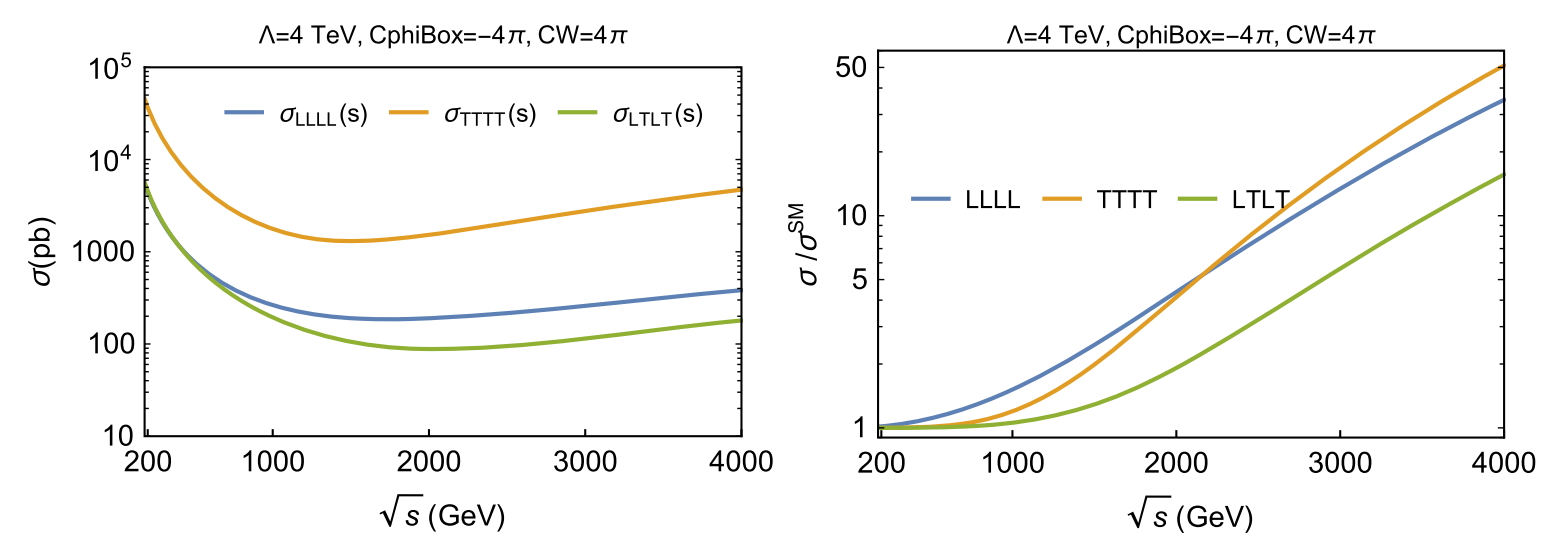

FIG. 3. Left: Polarized cross sections for the three mode cross sections (A26)-(A28) as a function of the c.m. energy $\sqrt{s}$. We have chosen as input values the coefficients shown at the top of the figures and $\theta_{\text {cut }}=\pi / 18$. Right: Similarly for the ratios of the polarized cross sections with respect to the SM result.

$$
\begin{aligned}
\sigma_{L L L L}(s) \approx & \frac{\bar{g}^{4}}{s}\left[\frac{A_{L}}{1-c^{2}}+B_{L}\left(\frac{C^{\varphi \square}}{\bar{g}^{2}}\right)\left(\frac{s}{\Lambda^{2}}\right)\right. \\
& \left.+\Gamma_{L}\left(\frac{C^{\varphi \square}}{\bar{g}^{2}}\right)^{2}\left(\frac{s}{\Lambda^{2}}\right)^{2}+\cdots\right],
\end{aligned}
$$

where $A_{i}, B_{i}, \Gamma_{i}(i=T, L)$ are dimensionless coefficients read from Eqs. (A26) and (A27) that depend upon the cutting angle $c \equiv \cos \left(\theta_{\text {cut }}\right)$ and ratios of vector-boson and Higgs masses, and "..." are corrections from higher than sixdimensional operators. The corresponding expression for $\sigma_{L T L T}$ and related cross sections are similar in form to Eq. (2.2). In order to clearly justify our points, we focus only on $C^{W}$ and $C^{\varphi \square}$ contributions in Eqs. (2.2)-(2.3). Full analytical expressions are given in Eqs. (A26)-(A28) of the Appendix.

In a weakly coupled UV theory with perturbative decoupling, dimensional analysis [44] results in $C^{W} \approx g^{3} /(4 \pi)^{2}, C^{\varphi \square} \approx g^{2}$. These effects could be important only if $A_{L} \approx\left(1-c^{2}\right) B_{L}\left(s / \Lambda^{2}\right)$ which is never the case for $c \approx 1$ and $s<\Lambda^{2}$, which suggests weak sensitivity to the small coupling regime in the $W^{+} W^{+}$process. This result, however, does strongly depend on the cutting angle $\theta_{\text {cut }}$. For example, in differential cross section distributions for the $L L L L$ mode, there are zeros for different values of SM EFT $C^{\varphi \square}$ and/or $C^{\varphi D}$ input values! This case study is however statistics limited at the LHC mainly because the SM dominant TTTT mode will not be affected, but may be important for future HL-LHC studies.

In a strongly coupled UV theory or in a UV theory with composite gauge bosons the loop suppression of $C^{W}$ may not be dictated by gauge invariance. Naive dimensional analysis [45] in such an extreme case may result in SM EFT coefficients as big as $C^{W} \approx 4 \pi$ and $C^{\varphi \square} \approx(4 \pi)^{2}$. What is most important here is the fact that the prefactors in Eq. (2.2), $\Gamma_{T}=36 \sqrt{2} / \pi$ and $A_{T}=64 / \pi$, are quite big and of the same order of magnitude. This guarantees large cross sections and visible effects in SM EFT. For example, if $C^{W}=C^{\varphi \square} \gg 1$, then the TTTT mode is again bigger than the $L L L L$ mode cross section by a factor of $g^{2} \Gamma_{T} / \Gamma_{L}=144 \sqrt{2}\left(G_{F} m_{W}^{2}\right) \simeq 15$. In this case, only contributions with $\operatorname{dim} 6^{2}$ in Eqs. (2.2)-(2.3) are dominant, an approximation which is independent of the cutting angle $\theta_{\text {cut }}$.

We demonstrate these effects in Fig. 3. We have chosen $\Lambda=4 \mathrm{TeV}$ and two nonzero Wilson coefficients: $C^{W}=4 \pi$ which affects only the TTTT and LTLT modes, and $C^{\varphi \square}=-4 \pi$ which affects only the $L L L L$ mode. ${ }^{7}$ The set of input values, although indicative, are consistent with the bounds from diboson production and electroweak fits [28] (see below) when marginalized over other coefficients. Obviously, the appearance of factors $\left(s / \Lambda^{2}\right)^{n}, n=1,2$ in Eqs. (A26)-(A28) make the cross sections rise. As explained, the dominant mode is $\sigma_{T T T T}$, i.e., the effect of $C^{W}$ seems the most promising. The steep rise of the $L L L L$ cross section for low $\sqrt{s}$ is due to the interference of SM and new physics arising from dimension-six operators. From the right panel of Fig. 3 it is obvious that the TTTT and $L L L L$ cross sections are enhanced by a factor of $\sim 10$ long before the EFT validity upper bound, ${ }^{8} s \approx \Lambda^{2}$, which encourages us to proceed to a more realistic VBS analysis at the LHC. We do this in the next section.

Furthermore, in the case of transverse gauge boson scattering, we consider the effect of dimension-eight operators. It is well known $[46,47]$ that dimension-eight operators may dominate (up to cancellations between coefficients induced by the UV theory) the VBS cross sections at energies

\footnotetext{
${ }^{7}$ The minus sign in $C^{\varphi \square}$ results in constructive interference.

${ }^{8}$ Tree-level unitarity bounds are discussed in detail in the next section.
} 


$$
\frac{s}{\Lambda^{2}} \gg \frac{\bar{g}}{g_{*}}
$$

Here we consider the case of strongly coupled (or composite) transverse polarizations of $W$ 's in the so-called Remedios models of Ref. [46]. In these models, $C^{W} \sim g_{*}$ and $C^{t 0, t 1, t 2, t 10} \sim g_{*}^{2}$, with the latter being the Wilson coefficients of dimension-eight operators defined in Appendix. There, we calculate all dominant helicity amplitudes with leading- $s$ and $s^{2}$ behavior. All but one independent helicity amplitude are masked by the $t$-channel photon and $Z$-boson exchange from the SM. The exception is the helicity-violating amplitude $\mathcal{M}_{++--}$(or $\mathcal{M}_{--++}$). When the coupling $g_{*}$ of the integrated UV theory is strong, i.e., much larger than the $S U(2)$ gauge coupling $\bar{g}$, these amplitudes give a large and positive contribution to the cross section. We therefore conclude that the inclusion of only the dimension-six operator $C^{W}$ is a robust conservative limit to $W^{+} W^{+}$ VBS. Notice also that (dimension-six) ${ }^{2}$ contributions in the amplitude come with opposite sign to the dimensioneight contributions when positivity constraints [48-50] are taken into account, which improves the EFT convergence. The situation is clearly explained in Fig. 4.

It is remarkable that in the case of Remedios-like scenarios the estimate on Wilson coefficients, based on power counting as given in Ref. [46], does not allow for fulfilment of positivity bounds. This suggests that positivity constraints [Eqs. (6.7)-(6.12) of Ref. [49]] constitute a significant limitation to models with composite dynamics of transverse electroweak modes. Notice however that these conditions can still be satisfied if, e.g., departures from the power-counting estimate are present in the strongly coupled regime, such as $C^{t 0}=C^{t 1}=$ $C^{t 10}=\left|C^{W}\right|^{2}=g_{*}^{2}$ and $C^{t 2} \geq 5 g_{*}^{2}$.

\section{THE REALISTIC STUDY: $p p \rightarrow j j W^{+} W^{+}$AT THE LHC}

In this section we describe the steps of our analysis and present numerical results relying on Monte Carlo simulations. The reaction in question is $p p \rightarrow j j W^{+} W^{+} \rightarrow$ $j j l l^{\prime} \nu_{l} \nu_{l^{\prime}}$, where $l=e^{+}, \mu^{+}$. The main goal is to estimate possible effects in same-sign $W W$ scattering in the HLLHC setup via the EFT approach. We analyze in an uncorrelated way one operator at a time while setting all of the remaining Wilson coefficients to zero. Strictly speaking, the above choice implicitly assumes a certain subset of BSM scenarios where it is valid. Indeed, it

\footnotetext{
${ }^{9}$ See Ref. [51] for an interesting example in which the growth with energy, although substantial, does not lead to limitations due to pure EFT convergence. Cases studied are operators that contribute to neutral anomalous TGC. Such operators start at dimension eight in SM EFT. Interestingly, the identified observables at $e^{+} e^{-}$colliders feature (dimension-eight) ${ }^{2}$ term suppression, leading to a "genuine" dimension-eight analysis.
}

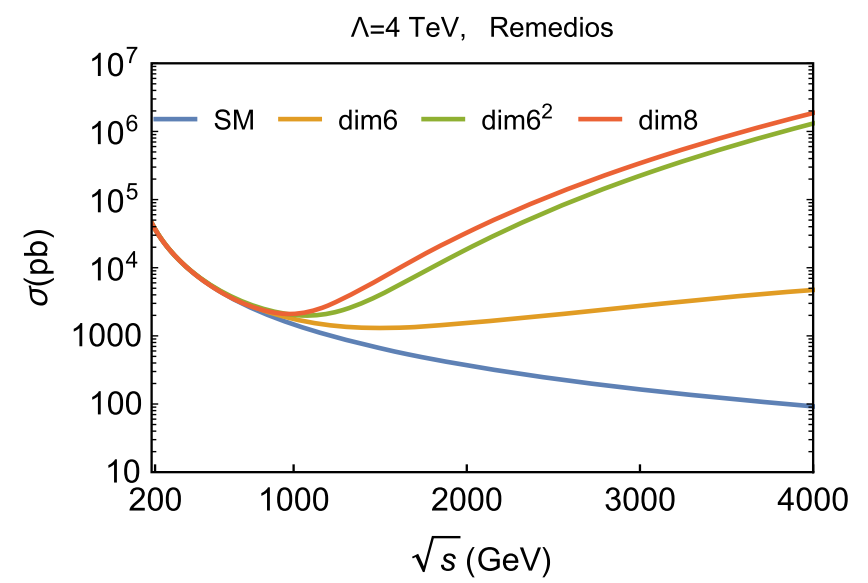

FIG. 4. Effects of (dimension-six $)^{2}$ and dimension-eight operator contributions for $C^{W}=g_{*}, C^{t 0, t 1, t 2, t 10}=g_{*}^{2}$, with $g_{*}=4 \pi$ in the so-called Remedios model for $W^{+} W^{+}$elastic scattering. The curves show the transversely polarized cross section $\sigma_{T T T T}$ starting from the SM (lower curve), and ending at the full $\mathrm{SM}+\operatorname{dim} 6+\operatorname{dim} 6^{2}+\operatorname{dim} 8$ (upper curve) helicity amplitudes. In Remedios the cross section cannot be smaller than the dim6 curve (orange curve).

constitutes a realistic assumption, e.g., (a) in the case of certain universal models with only bosonic weakly coupled BSM sector [52,53] (given the current experimental constraints on EFT coefficients), and (b) scenarios in which transversal modes of vector bosons have composite origin $[46,53]$. Nevertheless, the only nontrivial aspect that the above simplification misses is interference terms between different operators. Given the fact that different operators interfere only if they modify the same helicity amplitudes [54], the interference is significantly limited in our setupsee Eqs. (A17)-(A25) of the Appendix.

Below we focus on the largest possible deviations, using as our "benchmarks" the experimentally allowed boundary values for each $f_{i}$ in Eq. (1.1). We take as the source both the individual-operator-at-a-time limits and those from the global fit as they can be considered complementary within certain scenarios of new physics. In the latter case, correlations between different operators are in principle important. We comment on the validity of our oneoperator-at-a-time analysis below. Likewise, we also discuss the effects of background EFT operators.

\section{A. Perturbative unitarity bounds and unitarization issues}

The EFT operators in Table I induce $W W \rightarrow W W$ amplitude growth [see Eqs. (A17)-(A25)], which ultimately leads to violation of probability conservation at some energy scale $\sqrt{s^{U}}$, a certain $W W$ pair invariant mass, the latter being a function of $f_{i}$. In principle, it may happen that $\sqrt{s^{U}}$ is within the accessible range of $W W$ mass, and in fact we find that this is sometimes the case. 
Since predictions of the EFT amplitudes are ill defined above $\sqrt{s^{U}}$, the issue has to be addressed in some way in the analysis. In this work we apply additional weights to events above $M_{W W}=\sqrt{s^{U}}$ in the original nonregularized samples generated with Monte Carlo simulations. The weights are in general operator dependent. The applied procedure is supposed to ensure that the total $W W$ scattering BSM cross sections after regularization behave like $1 / s$ for $M_{W W}>\sqrt{s^{U}}$, and so it approximates the principle of constant amplitude, at least after some averaging over the individual helicity combinations [55]. Our choice of unitarization is often referred to in the literature as the (helicity-averaged) "Kink" method. ${ }^{10} \mathrm{We}$ find the above-mentioned weight to be equal to $\left(\sqrt{s^{U}} / M_{W W}\right)^{3.5}$ for the operator $Q_{W}$ and $\left(\sqrt{s^{U}} / M_{W W}\right)^{1.5}$ for $Q_{\varphi \square}$ and $Q_{\varphi W} \cdot{ }^{11}$ We refer to such a unitarized signal estimate as the total BSM signal.

Technically, $\sqrt{s^{U}}$ is determined by using the perturbative (tree-level) partial-wave unitarity condition [57,58]. The statement regarding $s^{U}$ from this condition is that for energies $s>s^{U}$, perturbativity of the EFT necessarily breaks down. In more detail, the unitarity limit has been determined by studying all helicity combinations for both $W^{+} W^{+}$and $W^{+} W^{-}$elastic scattering amplitudes. ${ }^{12}$ For each helicity amplitude, the first nonvanishing partial wave $\mathcal{T}^{(J)}$ (where always $J=0,1$, or 2) is identified and the unitarity bound is found. The scale where unitarity is violated, $\sqrt{s^{U}}$, is then identified as the lowest value among all such bounds. More explicitly, at tree level the condition reads (for a detailed discussion, see Refs. $[59,60])$

$$
\sqrt{N_{\lambda_{a} \lambda_{b}} N_{\lambda_{1} \lambda_{2}}}\left|\mathcal{T}_{\lambda_{a} \lambda_{b} ; \lambda_{1} \lambda_{2}}^{(J)}(s)\right| \leq \frac{1}{2}
$$

where the indices $\lambda_{a}, \lambda_{b}\left(\lambda_{1}, \lambda_{2}\right)$ denote outgoing (incoming) helicities, whereas $N_{x y}=1 / 2$ for identical particles, i.e., $x=y$, or otherwise $N_{x y}=1$. Then, the partialwave amplitudes $\mathcal{T}_{\lambda_{a} \lambda_{b} ; \lambda_{1} \lambda_{2}}^{(J)}(s)$ enter in the partial-wave expansion as

$$
\begin{aligned}
\mathcal{M}_{\lambda_{a} \lambda_{b} ; \lambda_{1} \lambda_{2}}= & 16 \pi \sum_{J=0}^{\infty}(2 J+1) \mathcal{T}_{\lambda_{a} \lambda_{b} ; \lambda_{1} \lambda_{2}}^{(J)}(s) \\
& \times D_{\lambda_{1}-\lambda_{2}, \lambda_{a}-\lambda_{b}}^{(J) *}\left(\Omega_{\mathbf{p}_{(a b)}}\right)
\end{aligned}
$$

\footnotetext{
${ }^{10}$ See Ref. [56] for a review and comparisons between several unitarization schemes.

${ }^{11}$ The different exponents follow from the fact that unitarity is first violated before the cross section gets dominated by its asymptotic terms.

${ }^{12}$ Both channels are governed by the same Wilson coefficients.
}

where $\Omega_{\mathbf{p}_{(a b)}}$ is a solid angle in the direction $\mathbf{p}_{(a b)}=\mathbf{p}_{a}+\mathbf{p}_{b}$, and $D_{m_{J}, \lambda}^{(J)}\left(\Omega_{\mathbf{p}}\right)$ are the Wigner functions satisfying the completeness relation

$$
\int d \Omega_{\mathbf{p}} D_{m_{J}^{\prime}, \lambda}^{\left(J^{\prime}\right)}\left(\Omega_{\mathbf{p}}\right) D_{m_{J}, \lambda}^{(J) *}\left(\Omega_{\mathbf{p}}\right)=\frac{4 \pi}{2 J+1} \delta_{J^{\prime} J} \delta_{m_{J}^{\prime} m_{J}}
$$

We analyze the terms that grow with energy $\left(\propto f_{i}\right)$, in which case no Coulomb singularity occurs and correspondingly no phase-space regularization has to be applied (e.g., a cut of $1 \mathrm{deg}$ in the forward and backward scattering regions). The results are cross-checked with VBFNLO 1.4.0 [61] for operators where direct applicability of the latter tool is possible in the context of the Warsaw basis, obtaining good agreement in the unitarity limits. When applied, the unitarity bound scale $\sqrt{s^{U}}$ is denoted by vertical lines in $M_{W W}$ distributions in figures below. Notice also that the unitarity bounds of VBS processes for dimension-six/-eight operators were presented in Refs. [62,63].

We would like to emphasize that within the EFT approach one does not have knowledge about what happens above $s^{U}$. There could be SM-like perturbative completion, or the theory could be nonperturbative. In the former case one expects $\sim 1 / s$, while in the latter $\sim(\log s)^{2}$, i.e., the saturation of the Froissart bound [64] for the asymptotic behavior of cross sections. The Froissart bound has the advantage of working in nonperturbative sense and might be more appropriate in the case of $Q_{W}$ treatment; the latter is currently poorly constrained. Applying the Froissart bound to the tail would enhance the signal, although only slightly, as we argue below; hence, our results are somewhat on the conservative side.

We verify that the bulk of the SM EFT effect is within the EFT-valid region, i.e., originates from the region $s<s^{U}$. To this aim, we define the "EFT-controlled" signal estimate [55] and compare it with the total BSM signal (the total BSM signal defined at the beginning of this section). The "EFT-controlled" signal estimate is calculated by replacing the generated high-mass tail $M_{W W}>\sqrt{s^{U}}$ with the one expected in the SM, while taking the EFT prediction for the region $M_{W W}<\sqrt{s^{U}}$. Hence, the "EFT-controlled" signal defines a signal originating uniquely from the operator within its (maximal) range of EFT validity. In turn, comparison between the total BSM signal estimate and the "EFT-controlled" signal allows for a verification of the significance of the tail region: the conclusions based on EFT are reliable only if the bulk of the BSM signal is in the "EFT-controlled" region [55]. In Sec. III. D we show that our results are approximately independent of how the contribution in the region above the unitarity bound $s>s^{U}$ is estimated, i.e., this issue should be a secondary effect; quantitatively, we check that the total BSM signal and the "EFT-controlled" estimate are statistically consistent within 
$2 \sigma$ for all of the operators and Wilson coefficients studied in Sec. III. D, with the exception of $f_{W}=1 \mathrm{TeV}^{-2}$ (discussed further therein).

Although it is well known that nonunitarized results do not directly have a physics interpretation, they could be safely taken as overestimated upper bounds on any unitarized results. For this reason, agreement between nonunitarized results and the "EFT-controlled" estimate implies little dependence on unitarization details. We make the corresponding comparison and observe consistency within $2 \sigma$ between nonunitarized results and the "EFTcontrolled" results for all of the cases (except $f_{W}=1 \mathrm{TeV}^{-2}$ ). Therefore, we choose to use the total BSM signal defined at the beginning of this section (popularly referred to as the "Kink" method) to obtain our central results throughout this work.

Note also that positivity bounds are not harmed by considering constant amplitudes for the asymptotic behavior of cross sections in our analysis.

\section{B. Method and kinematic variables}

For the following analysis two samples of $6 \times 10^{5}$ events consistent with the VBS topology for the process $p p \rightarrow j j \mu^{+} \mu^{+} \nu \nu$ are generated, each corresponding to a preselected arbitrary value of the $f_{W}$ or $f_{\varphi \square}$ coefficient, in MadGraph5_aMC@NLO [65] v2.6.2 at leading order at $14 \mathrm{TeV}$ $p p$ collision energy. Different $f_{i}$ values are obtained by applying weights to generated events, using the reweight command in MadGraph. The value $f_{i}=0, \forall i$ represents the SM predictions for each study. Results for the remaining relevant operators, i.e., $Q_{\varphi D}, Q_{\varphi W}$ are obtained using the reweight command with the $Q_{\varphi \square}$ sample. $^{13}$

The SmeftFR code [66] v2.01 (based on FeynRules [67]) is used to generate the UFO file [68] with an input parameter scheme $\left\{G_{F}, m_{W}, m_{Z}, m_{h}\right\}$, in SM EFT. Cross sections in the output of MadGraph are multiplied by a factor of 4 to account for all of the lepton combinations in the final state. Hadronization is done with PYTHIA v8.2 [69,70], run within MadGraph. Reconstruction level is generated via the MadAnalysis5 [71] v1.6.33 package (available within MadGraph). The FASTJET [72] v3.3.0 package is used with the jet clustering anti- $k_{T}$ algorithm with radius $=0.35$ and $\mathrm{ptmin}=20$. Finally, the detector efficiencies are set to $100 \%$.

The SM process $p p \rightarrow j j \ell^{+} \ell^{+} \nu \nu$ is treated as the irreducible background, while the "signal" is defined as the enhancement of the event yield relative to the SM prediction in the presence of a given operator $Q_{i}$. None of the reducible backgrounds are simulated. The reason is that reducible backgrounds (as we learned from, e.g., Fig. 3 Ref. [11]) roughly double the total statistics overall in the

\footnotetext{
${ }^{13}$ Samples for $Q_{W}$ were generated separately due to large reported uncertainties when reweighting from $Q_{\varphi \square}$.
}

VBS fiducial region and are mostly concentrated at low mass (both dilepton and dijet). Since VBS-related operators mainly modify the opposite end of the spectrum, reducible backgrounds probably will not be crucial to have an estimate of the possible effects. Yet another aspect is that additional operators might also modify the reducible backgrounds in some unforeseen ways. But such potential effects will need to be determined experimentally from other studies, in which those processes are not backgrounds but signals. We do not address this issue in our paper.

Following Ref. [55], the event selection criteria consist of requiring at least two reconstructed jets and exactly two leptons (muons or electrons) satisfying the following conditions: $M_{j j}>500 \mathrm{GeV}, \Delta \eta_{j j}>2.5, p_{T}^{j}>30 \mathrm{GeV}$, $\left|\eta_{j}\right|<5, p_{T}^{\ell}>25 \mathrm{GeV}$, and $\left|\eta_{\ell}\right|<2.5$, where $\eta_{j, \ell}$ is the pseudorapidity of jets $j$ or leptons $\ell$, respectively. The total $\mathrm{BSM}$ signal significances are computed as the square root of a $\chi^{2}$ resulting from a bin-by-bin comparison of the event yields in the binned distributions of different kinematic observables. Moreover, event distributions are always normalized to HL-LHC luminosity, i.e., $3000 \mathrm{fb}^{-1}$.

For each benchmark value of $f_{i=W, \varphi \square, \varphi D, \varphi W}$, the signal significance is assessed by studying the distributions of a large number of kinematic variables. These are

$$
\begin{aligned}
& m^{j j l l}, m^{l l}, m^{j j}, p_{T}^{j 1}, p_{T}^{j 2}, p_{T}^{l 1}, p_{T}^{l 2}, \\
& \eta^{j 1}, \eta^{j 2}, \eta^{l 1}, \eta^{l 2}, d \eta^{j}, d \phi^{j}, d \phi^{l}, \\
& R_{p_{T}} \equiv p_{T}^{l 1} p_{T}^{l 2} /\left(p_{T}^{j 1} p_{T}^{j 2}\right), \\
& M_{o 1} \equiv \sqrt{\left(\left|\vec{p}_{T}^{l 1}\right|+\left|\vec{p}_{T}^{l 2}\right|+\left|\vec{p}_{T}^{\text {miss }}\right|\right)^{2}-\left(\vec{p}_{T}^{l 1}+\vec{p}_{T}^{l 2}+\vec{p}_{T}^{\text {miss }}\right)^{2}}, \\
& M_{1 T}^{2} \equiv\left(\sqrt{\left(m^{l l}\right)^{2}+\left(\vec{p}^{l 1}\right)^{2}+\left(\vec{p}^{l 2}\right)^{2}}+\left|\vec{p}_{T}^{\text {miss }}\right|\right)^{2} \\
&-\left(\vec{p}^{l 1}+\vec{p}^{l 2}+\vec{p}_{T}^{\text {miss }}\right)^{2},
\end{aligned}
$$

where $l 1(2) \equiv$ (sub)leading lepton; $j 1(2) \equiv$ (sub)leading jet; $m$ is the invariant mass; $p_{T}^{\text {(miss) }} \equiv$ (missing) transverse momentum; $\eta \equiv$ pseudorapidity; $\phi \equiv$ azimuthal angle. Some of these variables are well known to be VBS blind, but in the context of a "background operator" they may still be useful. We find both angular variables $\eta$ and $\phi$ as well as $p_{T}$ 's involving jet(s) to be at most subleading in sensitivity. Moreover, all $p_{T}$ 's, $m$ 's, and $M$ 's are found to be at most subleading in sensitivity in the case of $Q_{\varphi \square}$ and $Q_{\varphi D}$. The most sensitive variables are $p_{T}^{l 1}$ for $Q_{W}, M_{o 1}$ for $Q_{\varphi W}$, and $R_{p_{T}}$ for $Q_{\varphi \square}, Q_{\varphi D}$.

We consider one-dimensional distributions of a single variable. Each distribution is divided into ten bins, arranged so that the Standard Model prediction in each bin is never lower than two events. Overflows were always included in the respective highest bins. Presented results and conclusions are always based upon the most sensitive variables, which is, in general, an operator-dependent outcome. 


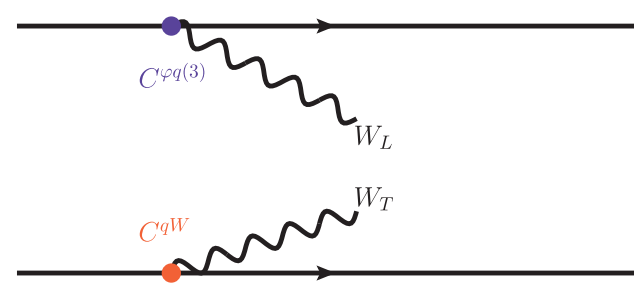

FIG. 5. Examples of background operators indicated by the associated Wilson coefficients.

The total BSM signal significance expressed in standard deviations $(\sigma)$ is defined as the square root of a $\chi^{2}$ resulting from comparing the bin-by-bin event yields:

$$
\chi^{2}=\sum_{i}\left(N_{i}^{\mathrm{BSM}}-N_{i}^{\mathrm{SM}}\right)^{2} / N_{i}^{\mathrm{SM}} .
$$

\section{Background operator analysis}

Before moving to the analysis of operators in Table I that modify the $W W \rightarrow W W$ reaction (VBS operators), we comment on the role of the dimension-six interactions that, although not affecting the subprocess $W W \rightarrow W W$ directly, may contribute at parton level to $p p \rightarrow j j l l^{\prime} \nu_{l} \nu_{l}^{\prime}$ (background operators), see Fig. 5. This is particularly relevant for the consistency of the discussion of potential effects of VBS operators when $f_{i}$ is constrained by the global fit, but also for a more complete description in general. We examine possible contributions (after the VBS cuts are applied) operator by operator, based on the bounds reported in Refs. [28,29] (non-four-fermion operators, the global, more permissive constraints are used in this work) and [30,31] (four-fermion operators) (with consistently applied flavor assumptions). The so-called "dipole" operators, i.e., $Q_{u W}, Q_{u B}, Q_{d G}, Q_{d W}, Q_{d B}$, are not present in the quoted references. They are however commonly claimed to be strongly constrained $[29,73]$. We checked that among these, only $Q_{u W}, Q_{d W}$, and $Q_{d G}$ would lead to noticeable effects for a representative $f_{i}=1 \mathrm{TeV}^{-2}$. Importantly, however, they also lead to commonly distinctive kinematic features compared to VBS operators, and hence they are in principle easily separated as part of "background" effects (discussed in detail below). We limit our examination to nonleptonic operators, because leptonic operators would contribute only at loop level to the process $p p \rightarrow j j W W$, and it is known that the on-shell projection of the outgoing $W$ 's can be defined in a gauge-invariant way and constitute a fine $(\lesssim 5 \%$ error) approximation [74,75]. Moreover, in this work we do not consider $C P$ - or $B$-violating operators. Out of the remaining, the coefficient corresponding to the operator $Q_{G}$ is known to be constrained at $O(0.01) \mathrm{TeV}^{-2}$ via multijet channels [76], whereas $Q_{\varphi}$ does not contribute to the tree-level amplitude. We also omit $Q_{\varphi G}$ as it is constrained by the process of Higgs boson production via gluon fusion at loop level [77]. In addition to this, we find no sensitivity to the four-fermion operators in the " $(\bar{L} R)(\bar{R} L)$ " and " $(\bar{L} R)(\bar{L} R)$ " classes even for generic BSM coupling in the strong-interaction regime. The experimental bounds on the remaining background operators are compiled in Table II. One can see that strong suppression is hidden in the Yukawa factors for the $Q_{u \varphi}, Q_{d \varphi}, Q_{u G}$ interactions (the minimal flavor violation hypothesis is assumed throughout the analysis). Since the light quarks play (by far) the leading role in our reaction, the Wilson coefficients are effectively of, at least, one-loop order in this case.

As far as non-four-fermion operators are concerned, we find that the current limits on all background dimension-six

TABLE II. Compilation of all of the experimental limits coming from global fits on dimension-six background operators used in this work, in $\mathrm{TeV}^{-2}$ units, and the maximal effect in standard deviations $(\sigma)$ in the $W^{+} W^{+}$ scattering process from each operator separately. Flavor assumptions are implicit and follow the references quoted. In particular, " $\times y$ " corresponds to the minimal flavor violation assumption.

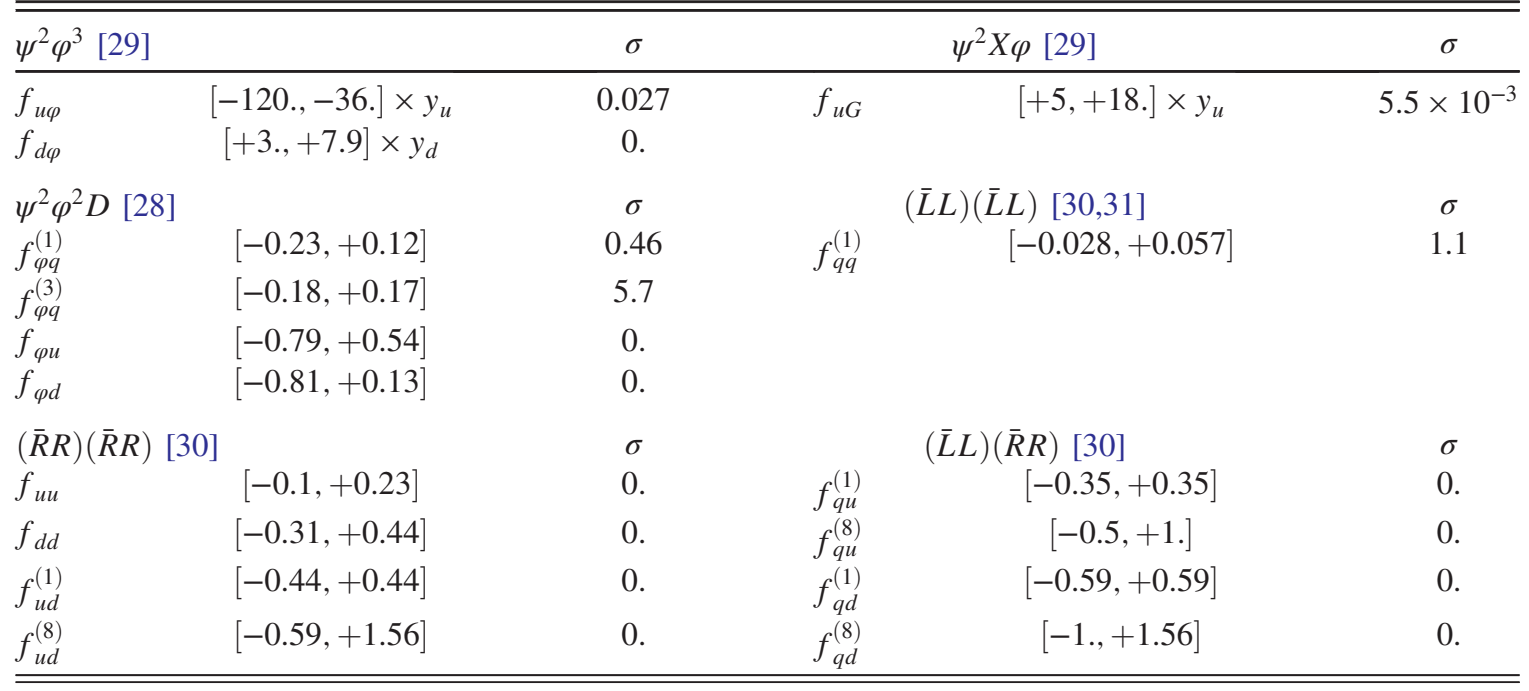


operators yield cross sections consistent with the SM prediction, with the exception of

$$
Q_{\varphi q}^{(3)}=\left(\varphi^{\dagger} i \stackrel{\leftrightarrow}{D}{ }_{\mu}^{I} \varphi\right)\left(\bar{q} \tau^{I} \gamma^{\mu} q\right)
$$

that yields a systematic discrepancy of around $\sim 5 \%$. Detailed analysis reveals that this operator gives a up to $\approx 5.5 \sigma$ potential discrepancy with SM at the HL-LHC. The distributions in $M_{W W}$ and $p_{T}^{l 1}$ are shown in Fig. 6 for $f_{\varphi q}^{(3)}=+0.17$ (the negative boundary value $f_{\varphi q}^{(3)}=-0.18$ is very similar).

One can observe that this background operator [see Fig. 5] has distinct dynamics compared to the VBS operators (presented subsequently). In particular, the contributions to $\chi^{2}$ are uniformly distributed among all of the bins $\left(p_{T}^{l 1}\right)$ and a similar feature holds for the $M_{W W}$ distribution. This is expected, because this background operator has nothing to do with the $W W$ mass, but as shown in Fig. 5 it mainly contributes to the hard scattering for longitudinal $W$ bosons, so the energy dependence measured in $W W$ processes should be different.

This shows that even when the background operator effect is large, it can be easily identified and disentangled by studying kinematic distributions: if one gets more than $5 \sigma$ between $Q_{\varphi q}^{(3)}$ and the SM, then one is also likely to get a $5 \sigma$ difference between the predicted shapes for $Q_{\varphi q}^{(3)}$ and any VBS operator. More quantitatively, we find, e.g., that $p_{T}^{j 1}$ is the most sensitive variable in the case of $Q_{\varphi q}^{(3) 14}$, while it is by far subleading in sensitivity for the VBS operators. Moreover, the weak sensitivity of jet $p_{T}$ 's also holds for the leading dimension-eight operators [78], which are usually considered as the leading effects in phenomenological/ experimental analyses for VBS processes so far. On the other hand, the highest sensitivity to $p_{T}^{j 1}$ also holds for the relevant dipole operators, as well as for the operator $Q_{\varphi u d}$, which is not studied in the "bounds" literature either; this constitutes the distinctive dynamics mentioned above for the dipole operators.

Concerning the potential effects in four-fermion dimension-six operators, we check that the limits reported in Ref. [30] were already sufficient to claim that the effects from these operators are at most negligible. The exception was the left current operator $Q_{q q}^{(1)}$ for which the search in the more up to date Ref. [31] was used to derive the factor by which the limits reported in Ref. [30] improve. We find the factor to be conservatively estimated by $\sim 3$, and using the improved constraints concluded that the operator might generate only negligible effects. Note that the other nonleptonic operator in

\footnotetext{
${ }^{14}$ Notice that it involves a contact interaction between two quarks and two vectors, so it contributes to a hard scattering of $\mathrm{q}$ and $\mathrm{W}$.
}

the $(L L)(L L)$ category, $Q_{q q}^{(3)}$, is identical to $Q_{q q}^{(1)}$ assuming flavor-diagonal Wilson coefficients.

\section{Main operator analysis}

From the previous subsection, we conclude that in practice it suffices to parametrize potential new physics (NP) effects from Wilson coefficients associated with the main dimension-six operators listed in Table I, i.e., the operators that modify the $W W \rightarrow W W$ subprocess. By saying this, we exclude from our discussion $C P$-violating operators $Q_{\tilde{W}}$ and $Q_{\varphi \tilde{W}}{ }^{15}$ for two reasons: first, their contribution in the cross section $W W \rightarrow W W$ is no different than the $C P$-conserving $Q_{W}$ and $Q_{\varphi W}$, and second, these operators are (usually) much more constrained than their $C P$-conserving cousins. Therefore, we focus on the CPC operators arranged in Table I for the numerical simulations to follow.

We begin by examining the possible effects at the HLLHC assuming the bounds on EFT coefficients come from the individual-operator-at-a-time analysis, based on the recent Ref. [28]; we quote the relevant constraints indicated as "individual" in Table III. The only $f_{i}$ upper-bounded by $O(0.1) \mathrm{TeV}^{-2}$ are $f_{\varphi \square}$ and $f_{W}$, and the remaining two $f_{\varphi D}$ and $f_{\varphi W}$ are bounded by $O(0.01) \mathrm{TeV}^{-2}$ (or stronger, depending on the sign) and give null discrepancies with the SM. In fact, at the boundaries $f_{\varphi \square} \approx \pm 0.5$ yields negligible effects as well. On the other hand, both boundaries on $f_{W}$ allow for large effects. The distributions $M_{W W}$ (left) and the total BSM signal in the most significant variable $p_{T}^{l 1}$ (right) are shown in Fig. 7 for $f_{W}=+0.36 \mathrm{TeV}^{-2}$ and in Fig. 8 for $f_{W}=-0.15 \mathrm{TeV}^{-2}$. Notice that in the right plots the total BSM signal is drawn in red and the "EFTcontrolled" signal in green, and that the red histogram is almost entirely covered by the green one, as anticipated in Sec. III. A.

Next, we consider bounds on $f_{i}$ from the global fit (indicated with "global" in Table III), again based on recent literature [28]. One can observe that constraints here are considerably more relaxed than the "individual" ones allowing for $f_{i}$ as large as $O\left(1\right.$.) $\mathrm{TeV}^{-2}$. It is due to correlations between various Wilson coefficients that contribute to the same observables used to determine the bounds. Therefore, such bounds could be understood as complementary and in principle saturated in UV models that normally allow for a plethora of dimension-six coefficients without too large hierarchies in their magnitudes, e.g., one can construct such models exploiting the UV vs tree-level matching dictionary [52] with a large number of different heavy species. As no correlation matrix was provided in Ref. [28], in what follows we

\footnotetext{
${ }^{15}$ The operator $Q_{\varphi \tilde{W} B}$ does not enter the leading amplitudes; see formulas in Appendix.
} 

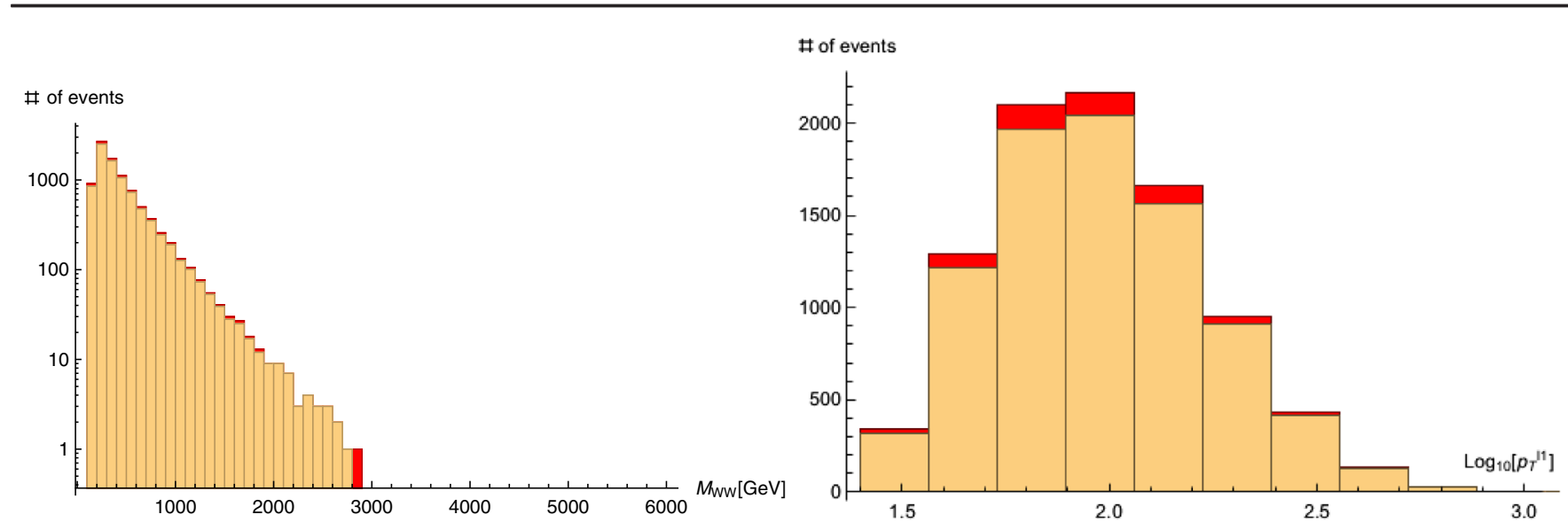

FIG. 6. Distributions in $W W$ system invariant mass $M_{W W}$ (left) and the most sensitive observable (here $p_{T}^{l 1}$ ) for $f_{\varphi q}^{(3)}=+0.17$ compared to the SM case. The BSM signal estimate is in red and the SM is in yellow.

examine the four VBS operators in a simplified way, i.e., one by one, and qualitatively describe the effect of correlations below.

We take an estimate on correlations from Ref. [29], which corresponds to similar physics assumptions. One can see that the correlations among the four relevant operators are mostly negligible. They are however somewhat mild between the following pairs: $\left(Q_{\varphi \square}, Q_{\varphi W}\right),\left(Q_{\varphi \square}, Q_{W}\right)$, and $\left(Q_{\varphi D}, Q_{W}\right)$. As we show in the Appendix, since the operators in each pair modify distinct helicities of the $W W \rightarrow W W$ subprocess, the corresponding effects do not interfere. As a result, to account for correlations corresponds to adding together the effects presented in this work of each operator, with appropriate weights from the correlation matrix. The most sensitive variables in Eq. (3.4) for each operator feature an increase in the number of events in the higher (relevant) bins independently of the sign of $f_{i}$. Moreover, notice that the account for mild correlation with $Q_{W}$ may affect the estimated effects because this operator is by far dominant in our analysis, and in this sense the results presented below for $Q_{\varphi \square}$ and $Q_{\varphi D}$ should be regarded as conservative ones.

The distributions in $M_{W W}$ together with the most sensitive variable are shown in Figs. 9-12 for the operators $Q_{\varphi D}, Q_{\varphi W}, Q_{\varphi \square}$, and $Q_{W}$, respectively (for $f_{i=\varphi D, \varphi W, \varphi \square}$ at the relevant boundary, either positive or negative).

As above, it is worth noticing that for all operators the bulk of the SM EFT effect is within the EFT-valid region. In terms of standard deviations $(\sigma)$, the maximal discrepancies for $Q_{\varphi D}, Q_{\varphi W}$, and $Q_{\varphi \square}$ at the HL-LHC allowed by current data are $4.4,15,12 \sigma$, respectively. Hence, these operators cannot in general be simply neglected in studies of HL-LHC prospects of discovery potential via the EFT approach.

The possible effects from $Q_{W}$ are, in accord with Sec. II, exceptionally large: the obtained discrepancy at the HLLHC is $O(100) \sigma$. In this case the exact number of $\sigma$ 's may vary depending on the chosen method of unitarization, as anticipated in Sec. III. A. For the scope of this paper it is rather inessential, so we report only the order of magnitude. Clearly, signal significance can be huge, far above $5 \sigma$ even in the "EFT-controlled" estimate, and therefore not at all negligible in VBS data analyses from the HL-LHC perspective. Moreover, a simple rescaling to current luminosity would imply large possible discrepancies already in the currently collected LHC data set. Though counterintuitive at first sight, it is partially a result of truncating the cross section consistently at dimension six, which (as already discussed in the Introduction) leads to conservative bounds from the diboson production channel. Although the samesign $W W$ scattering studies illustrate the necessity for better theoretical understanding (and as a consequence improvement) in setting constraints based on diboson production at the LHC, it also shows (i) the large potential in VBS processes to set constraints on the $f_{W}$ coefficient or, alternatively, (ii) by comparison of the red and green histograms in the right plots in Figs. 7 and 12, the large discovery potential which is characterizable by (seizable) "EFT triangle" [55]; interestingly, the EFT triangles for dimension-eight interactions are very limited $[54,55]$ due to EFT-validity issues caused by necessarily large high- $M_{W W}$ tails, which makes indirect searches in transversal $W W$ scattering via dimension six even more attractive.

TABLE III. Experimental constraints on the subset of operators modifying the process $W^{+} W^{+} \rightarrow W^{+} W^{+}$, based on the individual-operator-at-a-time or global marginalized fit analyses, from Ref. [28].

\begin{tabular}{lcccc}
\hline \hline & $f_{W}$ & $f_{\varphi \square}$ & $f_{\varphi D}$ & $f_{\varphi W}$ \\
\hline "individual" & {$[-0.15,+0.36]$} & {$[-0.44,+0.52]$} & {$[-0.025,+0.0015]$} & {$[-0.014,+0.0068]$} \\
"global" & {$[-1.3,+1.1]$} & {$[-3.4,+2.4]$} & {$[-2.7,+1.2]$} & {$[-0.14,+1.6]$} \\
\hline \hline
\end{tabular}




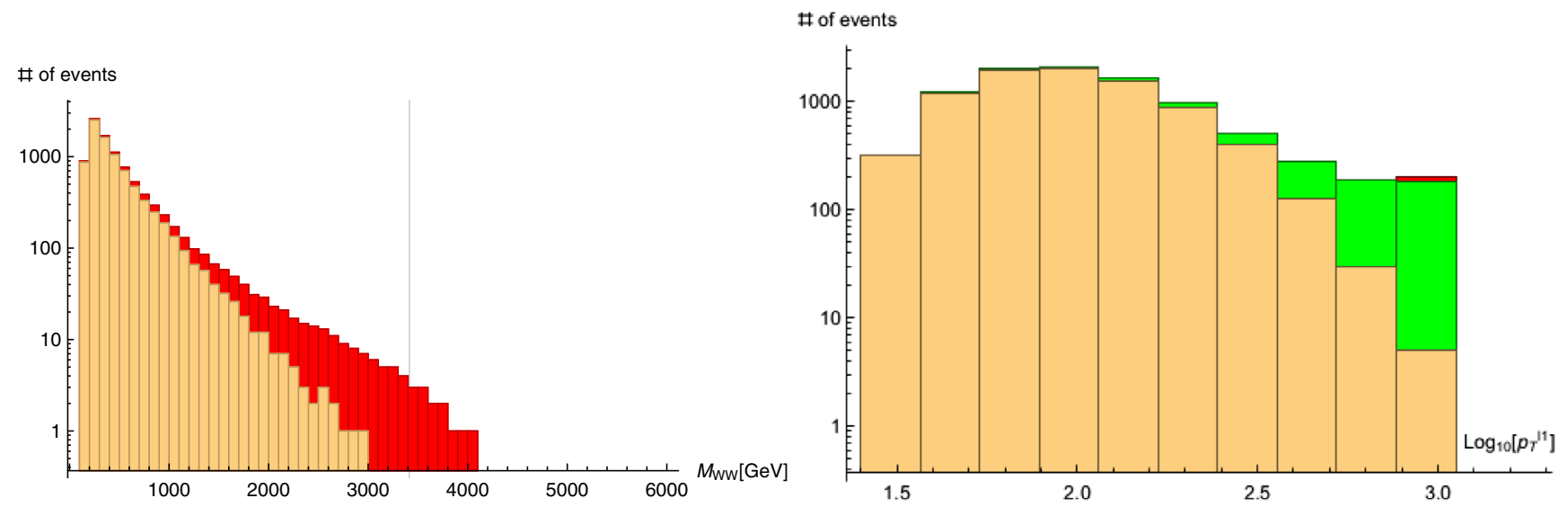

FIG. 7. Distributions in $W W$ pair invariant mass $M_{W W}$ (left) and the most sensitive observable (right, here $p_{T}^{l 1}$ ) for $f_{W}=+0.36 \mathrm{TeV}-2$ compared to the SM case. The total BSM signal estimate is in red and the SM is in yellow. Notice that in the right plot the "EFTcontrolled" signal estimate is shown in green (almost identical to the red one), normalized to the HL-LHC. The vertical line (left) denotes the scale above which the partial-wave unitarity condition is violated (see Sec. III. A).

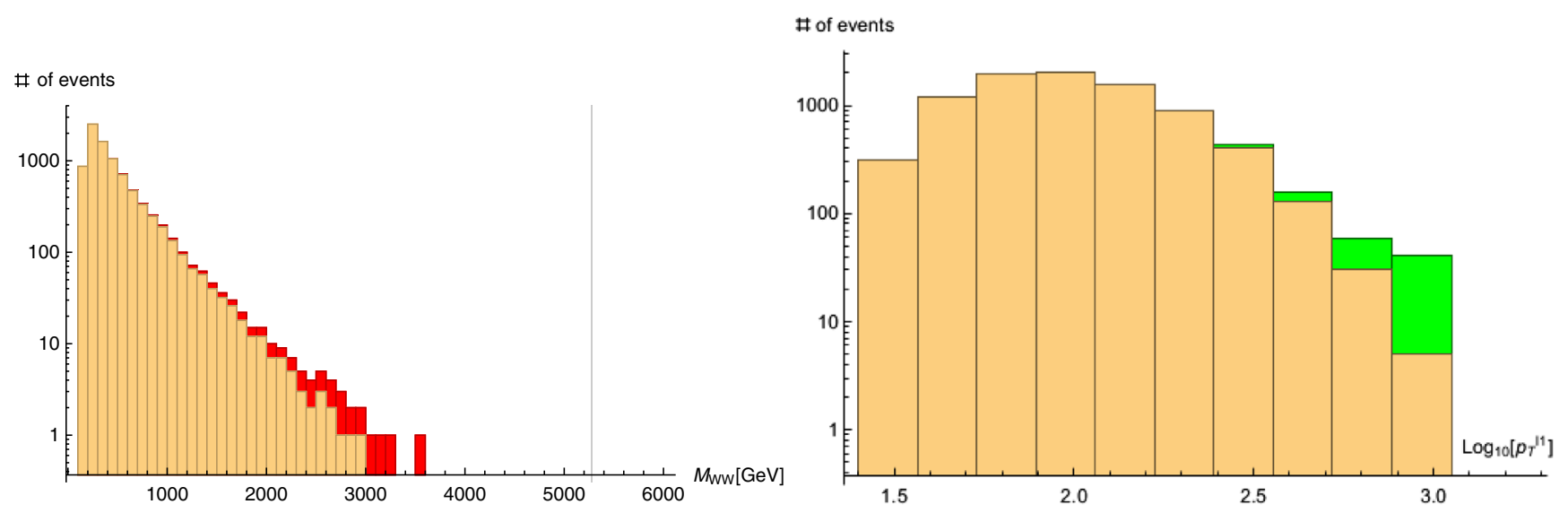

FIG. 8. Like Fig. 7 for $f_{W}=-0.15 \mathrm{TeV}^{-2}$.

\section{EPILOGUE}

In this article we performed a detailed study of SM EFT effects from dimension-six operators in a VBS process with like-sign- $W$ production proceeding to leptonic final states.

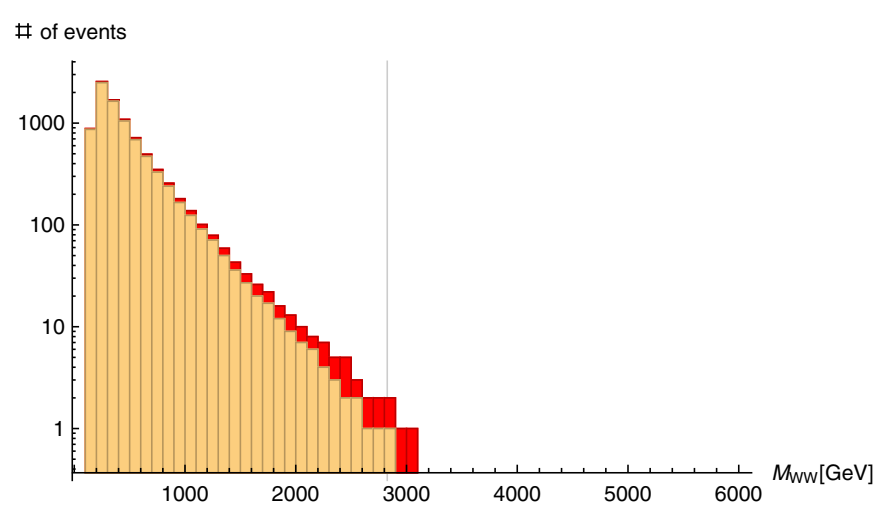

We studied all relevant dimension-six operators at tree level which are responsible for NP in VBS, including "background" operators responsible for NP in non-VBS parts of the full process $p p \rightarrow j j \ell \ell^{\prime} \nu \nu^{\prime}$. In addition, in our analysis

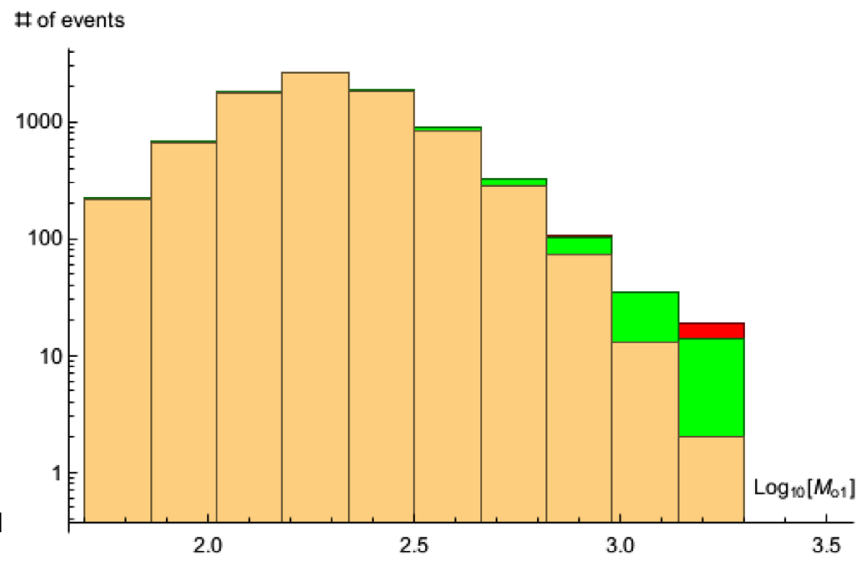

FIG. 9. Like in Fig. 7 for $f_{\varphi W}=+1.6 \mathrm{TeV}^{-2}$. 

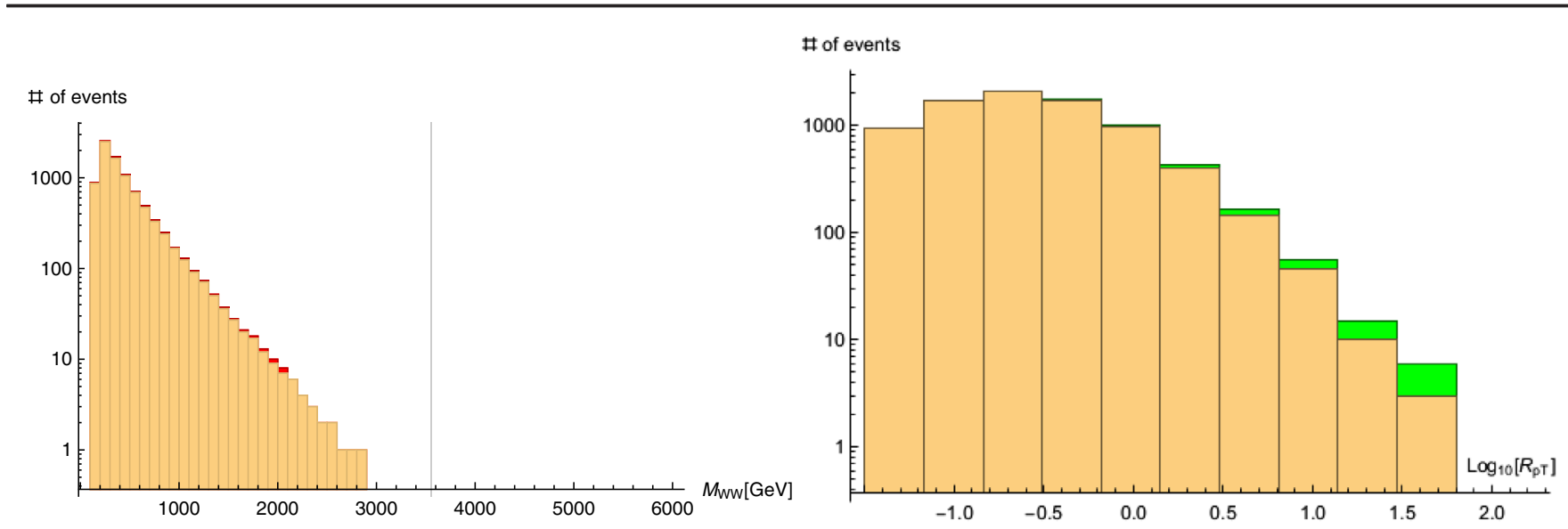

FIG. 10. Like in Fig. 7 for $f_{\varphi D}=-2.7 \mathrm{TeV}^{-2}$.

we exploited all available constraints on SM EFT Wilson coefficients and also derived the relevant perturbative unitarity bounds. The latter were applied to obtain a quantitative estimate on the role of high-energy tails that cannot be described within the EFT approach. We have presented useful analytical formulas which illustrate and support the robustness of our simulations' results.

We found that the role of dimension-six operators in modifying the $W^{+} W^{+} \rightarrow W^{+} W^{+}$process cannot be neglected in full generality, particularly for perspective
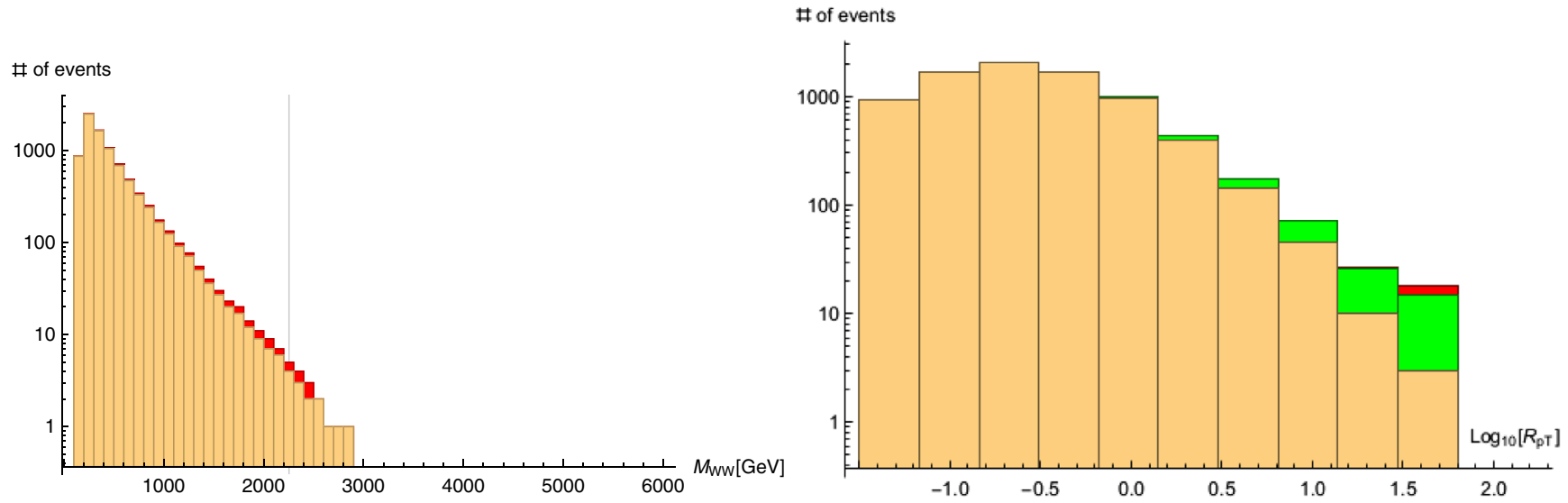

FIG. 11. Like in Fig. 7 for $f_{\varphi \square}=-3.4 \mathrm{TeV}^{-2}$.
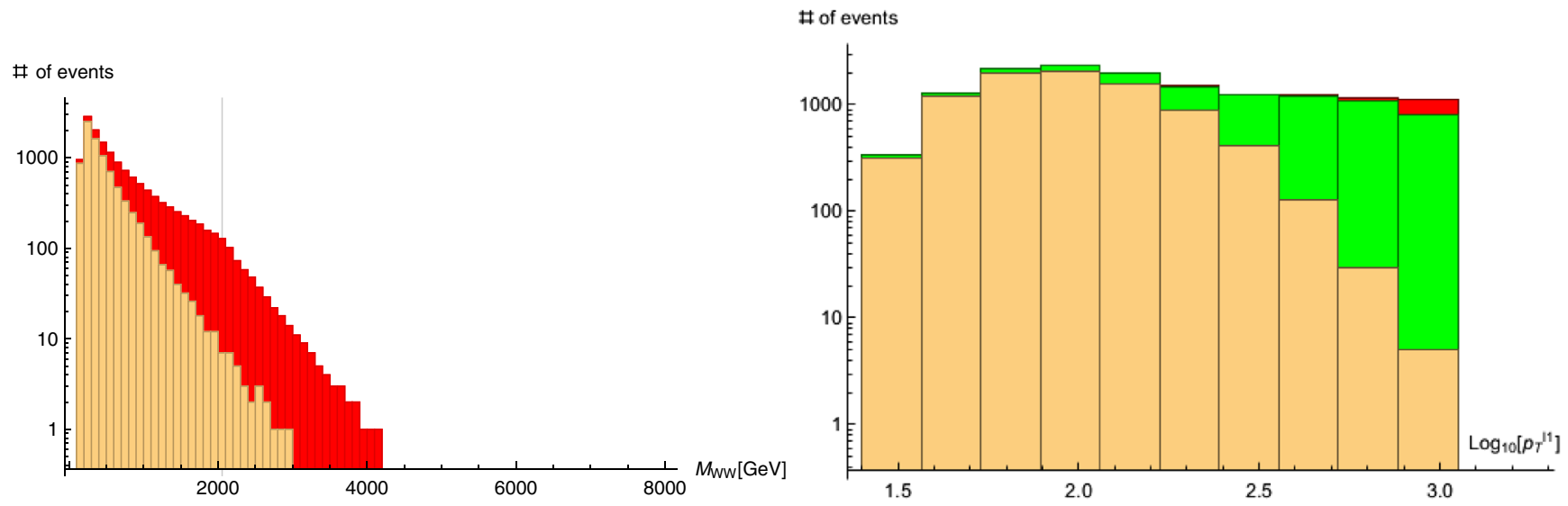

FIG. 12. Like in Fig. 7 for $f_{W}=+1 . \mathrm{TeV}^{-2}$. 
TABLE IV. Maximal effect estimates in standard deviations $(\sigma)$ for the relevant dimension-six interactions in same-sign $W W$ scattering allowed within present constraints as reported in the global fit analysis of Ref. [28], all normalized to the HL-LHC.

\begin{tabular}{lcccc}
\hline \hline & $\mathcal{O}_{W}$ & $\mathcal{O}_{\varphi \square}$ & $\mathcal{O}_{\varphi D}$ & $\mathcal{O}_{\varphi W}$ \\
\hline$\sigma^{\mathrm{HL}-\mathrm{LHC}} \leq$ & $\mathrm{O}(100)$. & 12. & 4.4 & 15. \\
\hline \hline
\end{tabular}

studies at the HL-LHC based on current constraints. This is particularly true when the constraints on dimension-six operators are taken from the global fit type analyses. The potential effect based on current global fit limits is summed up in Table IV for the relevant operators. It shows a particular sensitivity to strong dynamics for transverse $W$ polarization, the effects of which can be conservatively estimated with dimension-six truncated amplitudes. Plausibly, the background operators are already constrained strongly enough to claim that they play no role in $W W$ scattering process even from the HL-LHC perspective, with the exception of the $Q_{\varphi q}^{(3)}$ operator (and potentially $Q_{\varphi u d}$ and dipole operators). These VBS-polluting operators, however, feature very distinctive dynamics, and they can be separated by studying different kinematic distributions.

\section{ACKNOWLEDGMENTS}

We would like to thank Janusz Rosiek for his help in handling input parameter schemes in SmeftFR code. A. D. would like to thank Lambros Trifyllis and Kristaq Suxho for cross-checking a number of helicity amplitudes. P. K. is supported by the Spanish MINECO Project No. FPA201678220-C3-1- P (Fondos FEDER), A-FQM-211-UGR-18 (Junta de Andalucía, Fondos FEDER) and by National Science Centre, Poland, the PRELUDIUM project under Contract No. 2018/29/N/ST2/01153. The research of M. S. has been partly supported by VBSCan (COST action CA16108). We acknowledge partial support from National Science Centre, Poland, Grant No. DEC-2018/31/B/ ST2/02283.

\section{APPENDIX: HELICITY AMPLITUDES AND CROSS SECTIONS}

Probably the best way to write down the anatomy of a cross section, especially for vector-boson scattering, is to first calculate the helicity amplitudes for the given process. Being definitive, let us assume the elastic scattering of $W^{+}$ gauge bosons,

$$
W^{+}\left(p_{1}, \lambda_{1}\right)+W^{+}\left(p_{2}, \lambda_{2}\right) \rightarrow W^{+}\left(p_{3}, \lambda_{3}\right)+W^{+}\left(p_{4}, \lambda_{4}\right) .
$$

We consider the scattering plane to be the $x z$ plane with $\vec{p}_{1}$ in the $+\hat{z}$ direction, with the outgoing particle- 3 momentum $p_{3 x}$ in the positive $\hat{x}$ direction. More specifically, the kinematics are given by

$$
\begin{gathered}
p_{1}=(E, 0,0, p), \\
p_{2}=(E, 0,0,-p), \\
p_{3}=(E, p \sin \theta, 0, p \cos \theta), \\
p_{4}=(E,-p \sin \theta, 0,-p \cos \theta) .
\end{gathered}
$$

The center-of-mass scattering angle $\theta$ is restricted in the $\theta \in[0, \pi]$ interval. For process (A1) we have 81 helicity amplitudes. However, not all of them are distinct because of $C, P$, and $T$ transformations as well as the rotational invariance of the $S$-matrix. For example, the $C P T$ and $C P$ transformations read as

$C P T$ symmetry: $\mathcal{M}_{\lambda_{1}, \lambda_{2}, \lambda_{3}, \lambda_{4}}(\theta)=\mathcal{M}_{-\lambda_{3},-\lambda_{4},-\lambda_{1},-\lambda_{2}}(\theta)$,

$C P$ symmetry $: \mathcal{M}_{\lambda_{1}, \lambda_{2}, \lambda_{3}, \lambda_{4}}(\theta)=\mathcal{M}_{-\lambda_{2},-\lambda_{1},-\lambda_{4},-\lambda_{3}}(-\theta)$,

which relate the amplitudes up to a phase factor $[50,79]$.

For an elastic polarized cross section like $W^{+}\left(\lambda_{1}\right) W^{+}\left(\lambda_{2}\right) \rightarrow W^{+}\left(\lambda_{3}\right) W^{+}\left(\lambda_{4}\right)$ we have

$$
\left(\frac{d \sigma}{d \Omega}\right)_{\lambda_{1} \lambda_{2} \lambda_{3} \lambda_{4}}=\frac{1}{64 \pi^{2} s}\left|\mathcal{M}_{\lambda_{1} \lambda_{2} \lambda_{3} \lambda_{4}}(s, \cos \theta)\right|^{2},
$$

while for unpolarized ones we have to sum over final and initial helicities and average over initial helicities,

$$
\left(\frac{d \sigma}{d \Omega}\right)_{\text {unpolarized }}=\frac{1}{9} \sum_{\lambda_{1}, \lambda_{2}=-1}^{1} \sum_{\lambda_{3}, \lambda_{4}=-1}^{1}\left(\frac{d \sigma}{d \Omega}\right)_{\lambda_{1} \lambda_{2} \lambda_{3} \lambda_{4}} .
$$

The integrated cross section is defined as

$$
\sigma(s)=\int_{0}^{2 \pi} d \varphi \int_{\theta_{\text {cut }}}^{\pi-\theta_{\text {cut }}} d \theta \sin \theta \frac{d \sigma}{d \Omega},
$$

where $\theta_{\text {cut }}$ is an angular cut in scattering angle.

In Appendix we provide the helicity amplitudes $\mathcal{M}_{\lambda_{1} \lambda_{2} \lambda_{3} \lambda_{4}}$ in SM EFT with dimension-six operators by keeping the SM contributions to $O(1)$ and up to leading $O(s)$ amplitudes associated with the operators in Table I. In Appendix we also provide a partial list of amplitudes for SM EFT dimension-eight operators at leading $O\left(s^{2}\right)$ that relevant to our discussion. In calculating the amplitudes, we follow the notation of Ref. [36] for vertices, while we use SmeftFR [66] to output Feynman rules as input to FeynArts [80] and FormCalc [81] for amplitude calculations. In the 
case of longitudinal vector-boson scattering, we have checked our results analytically with the Goldstone boson equivalence theorem [37-43].

The SM leading $\mathcal{O}(1)$ contributions, where $\lambda$ is the Higgs self-coupling and $\bar{g}$ is the SM EFT improved $S U(2)_{L}$ gauge coupling, are

$$
\begin{gathered}
\mathcal{M}_{ \pm \pm \pm \pm}=-\frac{8 \bar{g}^{2}}{1-\cos ^{2} \theta} \\
\mathcal{M}_{ \pm \mp \mp \pm}=-2 \bar{g}^{2} \frac{(1-\cos \theta)}{(1+\cos \theta)} \\
\mathcal{M}_{ \pm \mp \pm \mp}=-2 \bar{g}^{2} \frac{(1+\cos \theta)}{(1-\cos \theta)} \\
\mathcal{M}_{0 \pm 0 \pm}=\mathcal{M}_{ \pm 0 \pm 0}=-2 \bar{g}^{2}\left(\frac{1}{1-\cos \theta}\right) \\
\mathcal{M}_{ \pm 00 \pm}=\mathcal{M}_{0 \pm \pm 0}=-2 \bar{g}^{2}\left(\frac{1}{1+\cos \theta}\right) \\
\mathcal{M}_{0000}=\frac{1}{2}\left(\bar{g}^{2}+\bar{g}^{\prime 2}\right)\left(1-\frac{4}{\sin ^{2} \theta}\right)-2 \lambda .
\end{gathered}
$$

The SM EFT leading- $s$ contributions from $C P$-conserving couplings associated with operators in the CPC row in Table I are

$$
\begin{gathered}
\mathcal{M}_{ \pm \mp \mp \mp}=\mathcal{M}_{\mp \pm \mp \mp}=\mathcal{M}_{\mp \mp \pm \mp}=\mathcal{M}_{\mp \mp \mp \pm}=-6 \bar{g} C^{W} \frac{s}{\Lambda^{2}}, \\
\mathcal{M}_{ \pm \pm \mp \mp}=12 \bar{g} C^{W} \frac{s}{\Lambda^{2}}, \\
\mathcal{M}_{0 \pm 0 \mp}=\mathcal{M}_{ \pm 0 \mp 0} \\
=-\frac{3}{4} \bar{g} C^{W}(3+\cos \theta) \frac{s}{\Lambda^{2}}+C^{\varphi W}(1-\cos \theta) \frac{s}{\Lambda^{2}},
\end{gathered}
$$

\section{Helicity amplitudes for $W^{+}\left(\lambda_{1}\right) W^{+}\left(\lambda_{2}\right) \rightarrow$ $W^{+}\left(\lambda_{3}\right) W^{+}\left(\lambda_{4}\right)$ in SM EFT}

$$
\begin{aligned}
& \mathcal{M}_{0 \pm \mp 0}= \mathcal{M}_{ \pm 00 \mp} \\
&=\frac{3}{4} \bar{g} C^{W}(3-\cos \theta) \frac{s}{\Lambda^{2}}-C^{\varphi W}(1+\cos \theta) \frac{s}{\Lambda^{2}}, \\
& \mathcal{M}_{0000}=\left(2 C^{\varphi \square}+C^{\varphi D}\right) \frac{s}{\Lambda^{2}} .
\end{aligned}
$$

The SM EFT leading- $s$ contributions from $C P$-violating couplings associated with operators in the CPV row in Table I are

$$
\begin{aligned}
& \mathcal{M}_{++--}=-\mathcal{M}_{--++}=-12 \bar{g} C^{\tilde{W}} \frac{s}{\Lambda^{2}}, \quad(\mathrm{~A} 22) \\
\mathcal{M}_{+---}= & \mathcal{M}_{-+--}=-\mathcal{M}_{--+-}=-\mathcal{M}_{---+}=6 \bar{g} C^{\tilde{W}} \frac{s}{\Lambda^{2}} \\
= & -\mathcal{M}_{-+++}=-\mathcal{M}_{+-++}=\mathcal{M}_{++-+}=\mathcal{M}_{+++-}, \\
\mathcal{M}_{0+-0}= & \mathcal{M}_{+00-} \\
= & -\frac{3}{4} \bar{g} C^{\tilde{W}}(3-\cos \theta) \frac{s}{\Lambda^{2}}+C^{\varphi \tilde{W}}(1+\cos \theta) \frac{s}{\Lambda^{2}} \\
= & -\mathcal{M}_{0-+0}=-\mathcal{M}_{-00+}, \\
\mathcal{M}_{0+0-} & \mathcal{M}_{+0-0} \\
= & \frac{3}{4} \bar{g} C^{\tilde{W}}(3+\cos \theta) \frac{s}{\Lambda^{2}}-C^{\varphi \tilde{W}}(1-\cos \theta) \frac{s}{\Lambda^{2}} \\
= & -\mathcal{M}_{0-0+}=-\mathcal{M}_{-0+0} .
\end{aligned}
$$

The SM results for the amplitudes agree with Refs. [38,82]. We convert the set of parameters $\left\{\bar{g}, \bar{g}^{\prime}, \bar{v}, \lambda\right\}$ to a set of input observables $^{16}\left\{G_{F}, m_{W}, m_{Z}, m_{h}\right\}$ and write out analytically the cross sections in the c.m. frame for longitudinal and transverse gauge bosons, averaging over the initial helicities. We find at leading order in $s$

$$
\begin{aligned}
\sigma_{T T T T}(s)= & \frac{8}{\pi s}\left(G_{F} m_{W}^{2}\right)^{2} c\left(\frac{9-c^{2}}{1-c^{2}}\right) \\
& +\frac{36 \sqrt{2} c}{\pi s}\left(G_{F} m_{W}^{2}\right)\left(\left|C^{W}\right|^{2}+\left|C^{\tilde{W}}\right|^{2}\right)\left(\frac{s}{\Lambda^{2}}\right)^{2},
\end{aligned}
$$

$$
\begin{aligned}
\sigma_{L L L L}(s)= & \frac{1}{2 \pi s}\left(G_{F} m_{Z}^{2}\right)^{2}\left[c \frac{9-c^{2}}{1-c^{2}}-2\left(c+2 L_{c}\right) \frac{m_{h}^{2}}{m_{Z}^{2}}+\left(\frac{m_{h}^{2}}{m_{Z}^{2}}\right)^{2}\right]+\frac{\sqrt{2}}{4 \pi s}\left(G_{F} m_{Z}^{2}\right)\left(2 C^{\varphi \square}+C^{\varphi D}\right)\left(\frac{s}{\Lambda^{2}}\right)\left[\left(c+2 L_{c}\right)-c \frac{m_{h}^{2}}{m_{Z}^{2}}\right] \\
& +\frac{c}{16 \pi s}\left(\frac{s}{\Lambda^{2}}\right)^{2}\left(2 C^{\varphi \square}+C^{\varphi D}\right)^{2},
\end{aligned}
$$

\footnotetext{
${ }^{16}$ See, for example, Ref. [83] for the conversion.
} 


$$
\begin{aligned}
\sigma_{L T L T}(s)= & \sigma_{T L T L}(s)=\sigma_{T L L T}(s)=\sigma_{L T T L}(s)=\frac{8}{\pi s}\left(G_{F} m_{W}\right)^{2}\left(\frac{c}{1-c^{2}}\right) \\
& -\frac{2^{1 / 4}}{16 \pi s}\left(G_{F} m_{W}^{2}\right)^{1 / 2}\left(\frac{s}{\Lambda^{2}}\right)^{2} c\left(9-c^{2}\right) \Re e\left(C^{W} C^{\varphi W *}+C^{\tilde{W}} C^{\varphi \tilde{W} *}\right) \\
& +\frac{3}{16 \pi s}\left(\frac{s}{\Lambda^{2}}\right)^{2}\left[\frac{c\left(3+c^{2}\right)}{9}\left(\left|C^{\varphi W}\right|^{2}+\left|C^{\varphi \tilde{W}}\right|^{2}\right)+\frac{\sqrt{2} c\left(27+c^{2}\right)}{4}\left(G_{F} m_{W}^{2}\right)\left(\left|C^{W}\right|^{2}+\left|C^{\tilde{W}}\right|^{2}\right)\right]
\end{aligned}
$$

Since $\cos \theta \rightarrow 0$ is not attainable, we have used a cut at the small angle $\theta_{\text {cut }}$ and integrate over $\theta_{\text {cut }}$ to $\pi-\theta_{\text {cut }}$. In Eqs. (A26)-(A28) we used the abbreviations $c \equiv \cos \theta_{\text {cut }}$ and $L_{c} \equiv \log \left(\frac{1-c}{1+c}\right)$. Numerically, if $\theta_{\text {cut }}=\pi / 18$, then $\left(1-c^{2}\right)^{-1} \approx 33$ and $L_{c} \approx-5$, and therefore cross sections are dominated by angles close to the beam.

\section{Helicity amplitudes for pure transversal $W$ 's with up to $d=8$ operators}

One of the important outcomes of our analysis is that the VBS cross section is strongly affected by the operator $O_{W}=\epsilon^{I J K} W_{\mu \rho}^{I} W^{\rho \sigma, J} W_{\sigma}^{\mu, K}$ which enters in the transverse- $W$ four-point interactions. Since this operator is not affected by Higgs field redefinitions after EW symmetry breaking, the inclusion of pure transverse $W^{4}$ operators is straightforward. For pure interactions $W^{4}$ the basis for dimensioneight operators reads $[16,17,48,49]^{17}$

$$
\begin{aligned}
& O_{t 0}=\left(W_{\mu \nu}^{I} W^{\mu \nu, I}\right)\left(W_{\alpha \beta}^{J} W^{\alpha \beta, J}\right), \\
& O_{t 1}=\left(W_{\alpha \nu}^{I} W^{\mu \beta, I}\right)\left(W_{\mu \beta}^{J} W^{\alpha \nu, J}\right), \\
& O_{t 2}=\left(W_{\alpha \mu}^{I} W^{\mu \beta, I}\right)\left(W_{\beta \nu}^{J} W^{\nu \alpha, J}\right), \\
& O_{t 10}=\left(W_{\mu \nu}^{I} \tilde{W}^{\mu \nu, I}\right)\left(W_{\alpha \beta}^{J} \tilde{W}^{\alpha \beta, J}\right) .
\end{aligned}
$$

The leading-s, $C P$-even helicity amplitudes up to $1 / \Lambda^{4}$ are symbolically written as

\footnotetext{
${ }^{17}$ Our notation for dimension-eight operators follows closely the Warsaw basis article [15] where operators contain only gauge fields and are not weighted by gauge couplings. For compariso, our $C^{t_{i}}$ coefficients are $g^{4} / 4$ times those of Ref. [49].
}

$\mathcal{M}=\mathrm{SM}+\mathrm{SM} \cdot \operatorname{dim} 6+(\operatorname{dim} 6)^{2}+\mathrm{SM} \cdot \operatorname{dim} 8$,

and are found to be

$$
\begin{aligned}
\mathcal{M}_{ \pm \pm \pm \pm} & =-\frac{8 \bar{g}^{2}}{1-\cos ^{2} \theta}+4\left(2 C^{t 1}+C^{t 2}\right) \frac{s^{2}}{\Lambda^{4}}, \quad(\mathrm{~A} 34) \\
\mathcal{M}_{ \pm \mp \pm \mp}= & -2 \bar{g}^{2} \frac{1+\cos \theta}{1-\cos \theta}-\frac{9}{2}\left|C^{W}\right|^{2} \cos ^{2}\left(\frac{\theta}{2}\right)(3-\cos \theta) \frac{s^{2}}{\Lambda^{4}} \\
& +(\cos \theta+1)^{2}\left(2 C^{t 0}+C^{t 1}+C^{t 2}-2 C^{t 10}\right) \frac{s^{2}}{\Lambda^{4}}, \\
\mathcal{M}_{ \pm \pm \mp \mp} & 12 \bar{g} C^{W} \frac{s}{\Lambda^{2}}-\frac{9}{2}\left(3-\cos ^{2} \theta\right)\left|C^{W}\right|^{2} \frac{s^{2}}{\Lambda^{4}} \quad(\mathrm{~A} 35) \\
& +\left(1+\cos ^{2} \theta\right)\left(4 C^{t 0}+2 C^{t 1}+C^{t 2}+4 C^{t 10}\right) \frac{s^{2}}{\Lambda^{4}} \\
& +8 C^{t 1} \frac{s^{2}}{\Lambda^{4}}, \\
\mathcal{M}_{ \pm \mp \mp \mp}=-6 \bar{g} C^{W} \frac{s}{\Lambda^{2}} . & (\mathrm{A} 36)
\end{aligned}
$$

The multiplicities of the above helicity amplitudes into the transversely polarized cross section $\sigma_{T T T T}$ are $2: 4: 2: 8$, respectively. All other contributions not written explicitly in Eqs. (A34)-(A37), growing at most like $\left(s v^{2} / \Lambda^{4}\right)$, are neglected. It is amusing to note that $(\operatorname{dim} 6)^{2}$ terms do not involve gauge couplings (as they should) in the broken phase: they result from the sum of $Z$ and $\gamma$ tree diagrams with identical Lorentz structures (see $W W Z$ and $W W \gamma$ vertices in Ref. [36]). On the other hand, dimension-eight leading- $s$ contributions arise from contact terms only.
[1] S. Weinberg, A Model of Leptons, Phys. Rev. Lett. 19, 1264 (1967).

[2] S. Glashow, Partial symmetries of weak interactions, Nucl. Phys. B22, 579 (1961).
[3] A. Salam, in Proceedings of the Eighth Nobel Symposium, edited by N. Svartholm (Wiley, New York, 1968), p. 367.

[4] S. Weinberg, Effective gauge theories, Phys. Lett. 91B, 51 (1980). 
[5] C. G. Callan, Jr., S. R. Coleman, J. Wess, and B. Zumino, Structure of phenomenological Lagrangians. 2., Phys. Rev. 177, 2247 (1969).

[6] S. R. Coleman, J. Wess, and B. Zumino, Structure of phenomenological Lagrangians. 1., Phys. Rev. 177, 2239 (1969).

[7] D. R. Green, P. Meade, and M.-A. Pleier, Multiboson interactions at the LHC, Rev. Mod. Phys. 89, 035008 (2017).

[8] B. Biedermann, A. Denner, and M. Pellen, Large Electroweak Corrections to Vector-Boson Scattering at the Large Hadron Collider, Phys. Rev. Lett. 118, 261801 (2017).

[9] D. Buttazzo, D. Redigolo, F. Sala, and A. Tesi, Fusing vectors into scalars at high energy lepton colliders, J. High Energy Phys. 11 (2018) 144.

[10] A. Costantini, F. De Lillo, F. Maltoni, L. Mantani, O. Mattelaer, R. Ruiz, and X. Zhao, Vector boson fusion at multi-TeV muon colliders, J. High Energy Phys. 09 (2020) 080 .

[11] CMS collaboration, Measurements of production cross sections of WZ and same-sign WW boson pairs in association with two jets in proton-proton collisions at $\sqrt{s}=13 \mathrm{TeV}$, Phys. Lett. B 809, 135710 (2020).

[12] CMS collaboration, Observation of Electroweak Production of Same-Sign W Boson Pairs in the Two Jet and Two SameSign Lepton Final State in Proton-Proton Collisions at $\sqrt{s}=13$ TeV, Phys. Rev. Lett. 120, 081801 (2018).

[13] I. Brivio and M. Trott, The standard model as an effective field theory, Phys. Rep. 793, 1 (2019).

[14] T. Appelquist and J. Carazzone, Infrared singularities and massive fields, Phys. Rev. D 11, 2856 (1975).

[15] B. Grzadkowski, M. Iskrzynski, M. Misiak, and J. Rosiek, Dimension-six terms in the standard model Lagrangian, J. High Energy Phys. 10 (2010) 085.

[16] C. W. Murphy, Dimension-8 operators in the standard model effective field theory, J. High Energy Phys. 10 (2020) 174.

[17] H.-L. Li, Z. Ren, J. Shu, M.-L. Xiao, J.-H. Yu, and Y.-H. Zheng, Complete set of dimension- 8 operators in the standard model effective field theory, arXiv:2005.00008.

[18] J. Bagger, V. D. Barger, K.-M. Cheung, J. F. Gunion, T. Han, G. A. Ladinsky, R. Rosenfeld, and C.-P. Yuan, Strongly interacting W W system: Gold plated modes, Phys. Rev. D 49, 1246 (1994).

[19] D. Espriu and B. Yencho, Longitudinal WW scattering in light of the Higgs boson discovery, Phys. Rev. D 87, 055017 (2013).

[20] J. Chang, K. Cheung, C.-T. Lu, and T.-C. Yuan, WW scattering in the era of post-Higgs-boson discovery, Phys. Rev. D 87, 093005 (2013).

[21] W. Kilian, T. Ohl, J. Reuter, and M. Sekulla, High-energy vector boson scattering after the Higgs boson discovery, Phys. Rev. D 91, 096007 (2015).

[22] S. Brass, C. Fleper, W. Kilian, J. Reuter, and M. Sekulla, Transversal modes and Higgs bosons in electroweak vectorboson scattering at the LHC, Eur. Phys. J. C 78, 931 (2018).

[23] J. Baglio et al., VBSCan mid-term scientific meeting, in VBSCan mid-term scientific meeting (2020), https:// inspirehep.net/literature/1789399.

[24] C. Degrande, O. Eboli, B. Feigl, B. Jäger, W. Kilian, O. Mattelaer et al., Monte Carlo tools for studies of nonstandard electroweak gauge boson interactions in multi-boson processes: A Snowmass White Paper, in Community Summer
Study 2013: Snowmass on the Mississippi (2013), https:// inspirehep.net/literature/1256129.

[25] O. J. P. Éboli and M. C. Gonzalez-Garcia, Classifying the bosonic quartic couplings, Phys. Rev. D 93, 093013 (2016).

[26] M. Fabbrichesi, M. Pinamonti, A. Tonero, and A. Urbano, Vector boson scattering at the LHC: A study of the WW $\rightarrow$ WW channels with the Warsaw cut, Phys. Rev. D 93, 015004 (2016).

[27] R. Gomez-Ambrosio, Studies of dimension-six EFT effects in vector boson scattering, Eur. Phys. J. C 79, 389 (2019).

[28] S. Dawson, S. Homiller, and S. D. Lane, Putting standard model EFT fits to work, Phys. Rev. D 102, 055012 (2020).

[29] J. Ellis, C. W. Murphy, V. Sanz, and T. You, Updated global SMEFT fit to Higgs, diboson and electroweak data, J. High Energy Phys. 06 (2018) 146.

[30] O. Domenech, A. Pomarol, and J. Serra, Probing the SM with dijets at the LHC, Phys. Rev. D 85, 074030 (2012).

[31] CMS collaboration, Search for new physics with dijet angular distributions in proton-proton collisions at $\sqrt{s}=13 \mathrm{TeV}$, J. High Energy Phys. 07 (2017) 013.

[32] S. Alte, M. König, and W. Shepherd, Consistent searches for SMEFT effects in non-resonant dijet events, J. High Energy Phys. 01 (2018) 094.

[33] A. Falkowski, M. Gonzalez-Alonso, A. Greljo, D. Marzocca, and M. Son, Anomalous triple gauge couplings in the effective field theory approach at the LHC, J. High Energy Phys. 02 (2017) 115.

[34] ATLAS collaboration, Observation of Electroweak Production of a Same-Sign $W$ Boson Pair in Association with Two Jets in $p p$ Collisions at $\sqrt{s}=13 \mathrm{TeV}$ with the ATLAS Detector, Phys. Rev. Lett. 123, 161801 (2019).

[35] ATLAS collaboration, Observation of electroweak $W^{ \pm} Z$ boson pair production in association with two jets in $p p$ collisions at $\sqrt{s}=13 \mathrm{TeV}$ with the ATLAS detector, Phys. Lett. B 793, 469 (2019).

[36] A. Dedes, W. Materkowska, M. Paraskevas, J. Rosiek, and K. Suxho, Feynman rules for the standard model effective field theory in $\mathrm{R}_{\xi}$-gauges, J. High Energy Phys. 06 (2017) 143.

[37] J. M. Cornwall, D. N. Levin, and G. Tiktopoulos, Derivation of gauge invariance from high-energy unitarity bounds on the s matrix, Phys. Rev. D 10, 1145 (1974).

[38] C. E. Vayonakis, Born helicity amplitudes and crosssections in nonabelian gauge theories, Lett. Nuovo Cimento 17, 383 (1976).

[39] B. W. Lee, C. Quigg, and H. B. Thacker, Weak interactions at very high-energies: The role of the Higgs boson mass, Phys. Rev. D 16, 1519 (1977).

[40] M. S. Chanowitz and M. K. Gaillard, The TeV physics of strongly interacting W's and Z's, Nucl. Phys. B261, 379 (1985).

[41] G. J. Gounaris, R. Kogerler, and H. Neufeld, Relationship between longitudinally polarized vector bosons and their unphysical scalar partners, Phys. Rev. D 34, 3257 (1986).

[42] Y.-P. Yao and C. P. Yuan, Modification of the equivalence theorem due to loop corrections, Phys. Rev. D 38, 2237 (1988).

[43] J. Bagger and C. Schmidt, Equivalence theorem redux, Phys. Rev. D 41, 264.

[44] C. Arzt, M. B. Einhorn, and J. Wudka, Patterns of deviation from the standard model, Nucl. Phys. B433, 41 (1995). 
[45] B. Gavela, E. Jenkins, A. Manohar, and L. Merlo, Analysis of general power counting rules in effective field theory, Eur. Phys. J. C 76, 485 (2016).

[46] D. Liu, A. Pomarol, R. Rattazzi, and F. Riva, Patterns of strong coupling for LHC searches, J. High Energy Phys. 11 (2016) 141.

[47] A. Azatov, R. Contino, C. S. Machado, and F. Riva, Helicity selection rules and noninterference for BSM amplitudes, Phys. Rev. D 95, 065014 (2017).

[48] G. N. Remmen and N. L. Rodd, Consistency of the standard model effective field theory, J. High Energy Phys. 12 (2019) 032.

[49] K. Yamashita, C. Zhang, and S.-Y. Zhou, Elastic positivity vs extremal positivity bounds in SMEFT: A case study in transversal electroweak gauge-boson scatterings, J. High Energy Phys. 01 (2021) 095.

[50] C. de Rham, S. Melville, A. J. Tolley, and S.-Y. Zhou, UV complete me: Positivity bounds for particles with spin, J. High Energy Phys. 03 (2018) 011.

[51] J. Ellis, H.-J. He, and R.-Q. Xiao, Probing new physics in dimension-8 neutral gauge couplings at $e^{+} e^{-}$colliders, Sci. China Phys. Mech. Astron. 64, 221062 (2021).

[52] J. de Blas, J. Criado, M. Perez-Victoria, and J. Santiago, Effective description of general extensions of the standard model: The complete tree-level dictionary, J. High Energy Phys. 03 (2018) 109.

[53] D. Marzocca et al., BSM benchmarks for effective field theories in Higgs and electroweak physics, arXiv:2009 .01249 .

[54] G. Chaudhary, J. Kalinowski, M. Kaur, P. Kozów, K. Sandeep, M. Szleper, and S. Tkaczyk, EFT triangles in the same-sign $W W$ scattering process at the HL-LHC and HE-LHC, Eur. Phys. J. C 80, 181 (2020).

[55] J. Kalinowski, P. Kozów, S. Pokorski, J. Rosiek, M. Szleper, and S. Tkaczyk, Same-sign WW scattering at the LHC: Can we discover BSM effects before discovering new states?, Eur. Phys. J. C 78, 403 (2018).

[56] C. Garcia-Garcia, M. Herrero, and R. A. Morales, Unitarization effects in EFT predictions of WZ scattering at the LHC, Phys. Rev. D 100, 096003 (2019).

[57] M. Jacob and G. C. Wick, On the general theory of collisions for particles with spin, Ann. Phys. (N.Y.) 7, 404 (1959).

[58] V. D. Barger, K.-M. Cheung, T. Han, and R. J. N. Phillips, Strong $W^{+} W^{+}$scattering signals at $p p$ supercolliders, Phys. Rev. D 42, 3052 (1990).

[59] P. Chankowski, Lecture notes: "QFT as a theory of particlesS-matrix and the scattering theory", https://www.fuw.edu.pl/ $\sim$ chank/qftoei.html.

[60] P. Kozów, The W and Z scattering as a probe of physics beyond the standard model: Effective field theory approach, Ph.D. thesis, Warsaw U., 2019, arXiv:1908.07596.

[61] K. Arnold et al., vBFnlo: A parton level Monte Carlo for processes with electroweak bosons, Comput. Phys. Commun. 180, 1661 (2009).

[62] T. Corbett, O. J. P. Éboli, and M. C. Gonzalez-Garcia, Unitarity constraints on dimension-six operators, Phys. Rev. D 91, 035014 (2015).

[63] E. D.S. Almeida, O. Éboli, and M. Gonzalez-Garcia, Unitarity constraints on anomalous quartic couplings, Phys. Rev. D 101, 113003 (2020).
[64] M. Froissart, Asymptotic behavior and subtractions in the mandelstam representation, Phys. Rev. 123, 1053 (1961).

[65] J. Alwall, R. Frederix, S. Frixione, V. Hirschi, F. Maltoni, O. Mattelaer, H.-S. Shao, T. Stelzer, P. Torrielli, and M. Zaro, The automated computation of tree-level and next-toleading order differential cross sections, and their matching to parton shower simulations, J. High Energy Phys. 07 (2014) 079.

[66] A. Dedes, M. Paraskevas, J. Rosiek, K. Suxho, and L. Trifyllis, SmeftFR - Feynman rules generator for the standard model effective field theory, Comput. Phys. Commun. 247, 106931 (2020).

[67] A. Alloul, N. D. Christensen, C. Degrande, C. Duhr, and B. Fuks, FeynRules 2.0-A complete toolbox for tree-level phenomenology, Comput. Phys. Commun. 185, 2250 (2014).

[68] C. Degrande, C. Duhr, B. Fuks, D. Grellscheid, O. Mattelaer, and T. Reiter, UFO-The universal FeynRules output, Comput. Phys. Commun. 183, 1201 (2012).

[69] T. Sjostrand, S. Mrenna, and P.Z. Skands, PYTHIA 6.4 physics and manual, J. High Energy Phys. 05 (2006) 026.

[70] T. Sjostrand, S. Mrenna, and P. Z. Skands, A brief introduction to PYTHIA 8.1, Comput. Phys. Commun. 178, 852 (2008).

[71] E. Conte, B. Fuks, and G. Serret, MadAnalysis 5, a userfriendly framework for collider phenomenology, Comput. Phys. Commun. 184, 222 (2013).

[72] M. Cacciari, G. P. Salam, and G. Soyez, FASTJET user manual, Eur. Phys. J. C 72, 1896 (2012).

[73] A. Falkowski, Higgs basis: Proposal for an EFT basis choice for LHC HXSWG, http://cds.cern.ch/record/2001958? $\ln =\mathrm{pl}$.

[74] A. Ballestrero, E. Maina, and G. Pelliccioli, $W$ boson polarization in vector boson scattering at the LHC, J. High Energy Phys. 03 (2018) 170.

[75] A. Ballestrero, E. Maina, and G. Pelliccioli, Different polarization definitions in same-sign $W W$ scattering at the LHC, Phys. Lett. B 811, 135856 (2020).

[76] F. Krauss, S. Kuttimalai, and T. Plehn, LHC multijet events as a probe for anomalous dimension-six gluon interactions, Phys. Rev. D 95, 035024 (2017).

[77] A. Falkowski and D. Straub, Flavourful SMEFT likelihood for Higgs and electroweak data, J. High Energy Phys. 04 (2020) 066.

[78] P. Kozów, L. Merlo, S. Pokorski, and M. Szleper, Same-sign WW Scattering in the HEFT: Discoverability vs EFT validity, J. High Energy Phys. 07 (2019) 021.

[79] A. Denner and T. Hahn, Radiative corrections to $W^{+} W^{-} \rightarrow$ $W^{+} W^{-}$in the electroweak standard model, Nucl. Phys. B525, 27 (1998).

[80] T. Hahn, Generating Feynman diagrams and amplitudes with FeynArts 3, Comput. Phys. Commun. 140, 418 (2001).

[81] T. Hahn and M. Perez-Victoria, Automatized one loop calculations in four-dimensions and D-dimensions, Comput. Phys. Commun. 118, 153 (1999).

[82] J. Layssac, F. M. Renard, and G. J. Gounaris, Vector boson pair production at supercollider: Useful approximate helicity amplitudes, Z. Phys. C 62, 139 (1994).

[83] A. Dedes, K. Suxho, and L. Trifyllis, The decay $h \rightarrow Z \gamma$ in the standard-model effective field theory, J. High Energy Phys. 06 (2019) 115. 